\title{
Inflation Dynamics in a Small Open ECONOMY MODEL UNDER INFLATION Targeting: Some Evidence from Chile
}

\author{
Marco Del Negro \\ Federal Reserve Bank of New York \\ Frank Schorfheide \\ University of Pennsylvania
}

Following the influential work of Christiano, Eichenbaum, and Evans (2005) and Smets and Wouters (2003), many central banks are building and estimating dynamic stochastic general equilibrium (DSGE) models with nominal rigidities and are using them for policy analysis. This new generation of sticky price (and wage) models typically emphasizes that relative price distortions caused by firms' partial inability to respond to changes in the aggregate price level lead to an inefficient use of factor inputs and, in turn, to welfare losses. In such an environment, monetary policy can partially offset these relative price distortions by stabilizing aggregate inflation. The policy problem is more complicated in an open economy environment, because domestic price movements are tied to exchange rate and terms-of-trade movements.

DSGE models can be used at different stages of the policymaking process. If the structure of the theoretical model is enriched to the point at which the model is able to track historical time series, DSGE models can be used as a tool to generate multivariate macroeconomic forecasts. Monetary policy in these models is typically represented by an interest rate feedback rule, and the innovations in the policy rule

We thank the Central Bank of Chile, and Mauricio Calani in particular, for providing us with the data used in the empirical analysis. Schorfheide gratefully acknowledges financial support from the Central Bank of Chile, the Alfred P. Sloan Foundation, and the National Science Foundation. The views expressed in this paper do not necessarily reflect those of the Federal Reserve Bank of New York or the Federal Reserve System.

Monetary Policy under Uncertainty and Learning, edited by Klaus Schmidt-Hebbel and Carl E. Walsh, Santiago, Chile. (c) 2009 Central Bank of Chile. 
can be interpreted as modest, unanticipated changes in monetary policy. These impulse responses can then be used to determine, say, the interest rate change necessary to keep inflation rates near a target level over the next year or two. Finally, one can use DSGE models to qualitatively or quantitatively analyze more fundamental changes in monetary policy, such as inflation versus output targeting or fixed versus floating exchange rates.

An important concern in the use of DSGE models is that some of the cross-equation restrictions generated by the economic theory are misspecified. This misspecification potentially distorts forecasts as well as policy predictions. In a series of papers (Del Negro and Schorfheide, 2004, 2008b; Del Negro and others, 2007), we develop an econometric framework that allows us to gradually relax the crosscoefficient restrictions and construct an empirical model that can be regarded as a structural vector autoregression (VAR) and that retains many of the features of the underlying DSGE model, at least to the extent that they are not grossly inconsistent with historical time series. We refer to this empirical model as DSGE-VAR.

Based on a small open economy model developed by Galí and Monacelli (2005) and modified for estimation purposes by Lubik and Schorfheide (2007), we here present estimation results for such a DSGE-VAR model for the Chilean economy, using data on output growth, inflation, interest rates, exchange rates, and terms of trade. Throughout the 1990s, monetary policy was conducted within a partial inflation-targeting regime, since the monetary authorities were targeting the exchange rate in addition to inflation. Moreover, the inflation target was evolving during this period. In September 1999, Chile entered a floating exchange rate regime, thus adopting full-fledged inflation targeting. We therefore choose to use only post1999 data, which leaves a fairly short sample for the estimation of an empirical model for monetary policy analysis. An important advantage of the DSGE-VAR framework is that it allows us to estimate a vector autoregressive system with a short time series. Roughly speaking, this estimation augments actual observations by hypothetical observations, generated from a DSGE model, to determine the coefficients of the VAR. Over time, as more actual observations become available, our procedure will decrease or increase the fraction of actual observations in the combined sample, depending on whether the data contain evidence of model misspecification.

The empirical analysis is divided in four parts. We begin by estimating both the DSGE model and the DSGE-VAR. The DSGE-VAR 
produces estimates of the coefficients of the underlying theoretical model along with the VAR coefficients. Our discussion first focuses on the monetary policy rule estimates. Starting from a prior that implies a strong reaction of the Central Bank to inflation movements, we find that since 1999 the Central Bank has not reacted significantly to exchange rate or terms-of-trade movements, which is consistent with the official policy statements. In the second part, we study the fit of our small-scale DSGE model. As in our earlier work, the fit of the empirical vector autoregressive model can be improved by relaxing the theoretical cross-coefficient restrictions. More interestingly, due to the short sample size, the fraction of DSGE-model-generated observations in the mixed sample that is used for the estimation of the VAR is higher than, say, in estimations that we have conducted for the United States. Consequently, the dynamics of the DSGE-VAR closely resemble those of the underlying DSGE model, which is documented in the third part of the empirical analysis. Here, we focus specifically on how the various structural shocks affect inflation movements.

In the final part of the empirical analysis, we study the effect of changes in the monetary policy rule. Conceptually, this type of analysis is very challenging. If one believes that the DSGE model is not misspecified, then one can determine the behavioral responses of firms and households by re-solving the model under alternative policy rules. Empirical evidence of misspecification of cross-equation restrictions, however, raises questions about the reliability of the DSGE model's policy implications. In Del Negro and Schorfheide (2008b), we develop tools that allow us, under particular invariance assumptions, to check for the robustness of the DSGE model conclusions to the presence of misspecification. We apply some of these tools to explore what would happen to the variability of inflation if the Central Bank responded more or less to inflation as well as terms-of-trade movements.

A substantial amount of empirical literature explores the Chilean economy, including many of the issues analyzed in the paper: the specification of the policy rule, the dynamics of inflation, and the responses of domestic variables to external shocks. ${ }^{1}$ For most of this literature, the estimation period comprises the 1990s, a period of convergence toward full-fledged inflation targeting (see Mishkin and Schmidt-Hebbel, 2007). Because of concerns about structural change between the early phase of inflation targeting and the current one, we

1. See Chumacero (2005) and Céspedes and Soto (2007); these two papers also provide a survey of the existing literature. 
do not use the early period in the estimation. Our results, therefore, are not directly comparable with those of the previous literature. Caputo and Liendo (2005) present a very close paper to ours, in that they also estimate the Gali-Monacelli/Lubik-Schorfheide model on Chilean data. Most of their results include the 1990s, however, which makes comparisons hard. Furthermore, Caputo and Liendo (2005) use an estimate of the output gap as an observable, as opposed to the output growth rate used in this paper. They also perform subsample analysis, and one of their subsamples is close to the one used here. For that subsample, many of their results are similar to ours. Another close paper to ours is by Caputo, Liendo, and Medina (2007), who estimate a more sophisticated small open economy DSGE model using Bayesian methods on Chilean data. Again, their use of 1990s data makes the results not directly comparable. In future work it would be interesting to apply some of the techniques used in our paper to a larger-scale small open economy DSGE model.

The remainder of the paper is organized as follows. Section 1 contains a description of the small open economy model. The DSGEVAR framework developed in Del Negro and Schorfheide (2004, 2008b) is reviewed in section 2 . The data set used for the empirical analysis is discussed in section 3. Empirical results are summarized in section 4 , and section 5 concludes. Detailed derivations of the DSGE model are provided in the appendix.

\section{A Small Open Economy Model}

We now describe a simple small open economy DSGE model for the Chilean economy. The model has been previously estimated with data from Australia, Canada, New Zealand, and the United Kingdom in Lubik and Schorfheide (2007). It is a simplified version of the model developed by Galí and Monacelli (2005). We restrict our exposition to the key equilibrium conditions, represented in log-linearized form. ${ }^{2}$ Derivations of these equations are relegated to the appendix. All variables below are measured in percentage

2. We follow Galí and Monacelli (2005) and Lubik and Schorfheide (2007) in solving the detrended model by log-linearization around its steady state. The appendix describes the nonlinear equilibrium conditions and the log-linearization step. In the case of the Chilean economy, it is an open question whether log-linearization provides an accurate solution to the model, given that shocks are larger in size than in developed economies. We are aware of the issue, but at this stage our computational capabilities limit the extent to which we can use alternative solution methods. 
deviations from a stochastic balanced growth path, induced by a technology process, $Z_{t}$, that follows a first-order autoregressive, or $\mathrm{AR}(1)$, process in growth rates:

$\Delta \ln Z_{t}=\gamma+\hat{z}_{t}$

where $\hat{z}_{t}=\rho_{z} \hat{z}_{t-1}+\sigma_{z} \varepsilon_{z, t}$. Here, $\Delta$ denotes the temporal difference operator.

We begin with a characterization of monetary policy. We assume that monetary policy is described by an interest rate rule. The central bank adjusts its instrument in response to movements in consumer price index (CPI) inflation and output growth. Moreover, we allow for the possibility of including nominal exchange rate depreciation or terms-of-trade changes in the policy rule:

$$
\hat{R}_{t}=\rho_{R} \hat{R}_{t-1}+\left(1-\rho_{r}\right)\left[\psi_{1} \hat{\pi}_{t}+\psi_{2}\left(\Delta \hat{y}_{t}+\hat{z}_{t}\right)+\psi_{3} \Delta x_{t}\right]+\sigma_{r} \varepsilon_{r, t} .
$$

Since $\hat{y}_{t}$ measures percentage deviations from the stochastic trend induced by the productivity process $Z_{t}$, output growth deviations from the mean, $\gamma$, are given by $\Delta \hat{y}_{t}+\hat{z}_{t}$. We use $\Delta x_{t}$ to represent either exchange rate or terms-of-trade changes. To match the persistence in nominal interest rates, we include a smoothing term in the rule, with $0 \leq \rho_{r}<1$. Finally, $\varepsilon_{r, t}$ is an exogenous policy shock that can be interpreted as the nonsystematic component of monetary policy.

The household behavior in the home country is described by a consumption Euler equation in which we use equilibrium conditions to replace domestic consumption and CPI inflation by a function of domestic output, $\hat{y}_{t}$; output in the absence of nominal rigidities (potential output), $\bar{y}_{t}$; and inflation associated with domestically produced goods, $\hat{\pi}_{H, t}$ :

$$
\hat{y}_{t}-\bar{y}_{t}=E_{t}\left[\hat{y}_{t+1}-\bar{y}_{t+1}\right]-(\tau+\lambda)\left(\hat{R}_{t}-E_{t}\left[\hat{\pi}_{H, t+1}+\hat{z}_{t+1}\right]\right),
$$

where $E_{t}$ is the expectation operator and where

$$
\lambda=\alpha(2-\alpha)(1-\tau)
$$


and

$\bar{y}_{t}=-\frac{\lambda}{\tau} \hat{y}_{t}^{*}$

The parameter $0<\alpha<1$ represents the fraction of imported goods consumed by domestic households, $\tau$ is their intertemporal substitution elasticity, and $\hat{y}_{t}^{*}$ is an exogenous process that captures foreign output (relative to the level of total factor productivity). Notice that for $\alpha=0$, equation (3) reduces to its closed economy variant.

Optimal price setting by domestic firms leads to the following Phillips curve relationship:

$\hat{\pi}_{H, t}=\beta E_{t}\left[\hat{\pi}_{H, t+1}\right]+\kappa \widehat{m c}_{t}$,

where marginal costs can be expressed as

$\widehat{m c}_{t}=\frac{1}{\tau+\lambda}\left(\hat{y}_{t}-\bar{y}_{t}\right)$.

The slope coefficient $\kappa>0$ reflects the degree of price stickiness in the economy. As $\kappa \rightarrow \infty$, the nominal rigidities vanish.

We define the terms of trade, $\hat{Q}_{t}$, as the relative price of exports in terms of imports and let $\hat{q}_{t}=\Delta \hat{Q}_{t}$. The relationship between CPI inflation and $\hat{\pi}_{H, t}$ is given by

$\hat{\pi}_{t}=\hat{\pi}_{H, t}-\alpha \hat{q}_{t}$.

Assuming that relative purchasing power parity (PPP) holds, we can express the nominal exchange rate depreciation as

$\hat{e}_{t}=\hat{\pi}_{t}-\hat{\pi}_{t}^{*}-(1-\alpha) \hat{q}_{t}$,

where $\hat{\pi}_{t}^{*}$ is a world inflation shock that we treat as an unobservable. An alternative interpretation, as in Lubik and Schorfheide (2006), is that $\hat{\pi}_{t}^{*}$ captures misspecification or deviations from PPP. Since the other variables in the exchange rate equation are observed, this relaxes the potentially tight cross-equation restrictions embedded in the model. 
Equations (3) and (5) have been derived under the assumption of complete asset markets and perfect risk sharing, which implies that

$$
\hat{q}_{t}=-\frac{1}{\tau+\lambda}\left(\Delta \hat{y}_{t}-\Delta \hat{y}_{t}^{*}\right)
$$

This equilibrium condition clearly indicates that the terms of trade are endogenous in the model, because domestic producers have market power. Instead of imposing this condition, however, we follow the approach in Lubik and Schorfheide (2007) and specify an exogenous law of motion for the terms-of-trade movements:

$$
\hat{q}_{t}=\rho_{q} \hat{q}_{t-1}+\sigma_{q} \varepsilon_{q, t}
$$

In the empirical section, we provide evidence on the extent to which this assumption is supported by the data.

Equations (2) to (8) form a rational expectations system that determines the law of motion for domestic output, $\hat{y}_{t}$; flexible price output, $\bar{y}_{t}$; marginal costs $\widehat{m c}_{t}$; CPI inflation, $\hat{\pi}_{t}$; domestically produced goods inflation, $\hat{\pi}_{H, t}$; interest rates, $\hat{R}_{t}$; and nominal exchange rate depreciations, $\hat{e}_{t}$. We treat monetary policy shocks, $\varepsilon_{r, t}$, technology growth, $\hat{z}_{t}$, and terms-of-trade changes, $\hat{q}_{t}$, as exogenous. Moreover, we assume that rest-of-the-world output and inflation, $\hat{y}_{t}^{*}$ and $\hat{\pi}_{t}^{*}$, follow exogenous autoregressive processes:

$$
\begin{aligned}
& \hat{\pi}_{t}^{*}=\rho_{\pi^{*}} \hat{\pi}_{t-1}^{*}+\sigma_{\pi^{*}} \varepsilon_{\pi^{*}, t} ; \\
& \hat{y}_{t}^{*}=\rho_{y^{*}} \hat{y}_{t-1}^{*}+\sigma_{y^{*}} \varepsilon_{y^{*}, t},
\end{aligned}
$$

The rational expectations model described by equations (1) to (11) can be solved with standard techniques, such as Sims (2002). We collect the DSGE model parameter in the vector $\boldsymbol{\theta}$, defined as

$\boldsymbol{\theta}=\left[\psi_{1}, \psi_{2}, \psi_{3}, \rho_{r}, \alpha, \beta, \tau, \rho_{z}, \rho_{q}, \rho_{\pi^{*}}, \rho_{y^{*}}, \sigma_{r}, \sigma_{z}, \sigma_{q}, \sigma_{\pi^{*}}, \sigma_{y^{*}}\right]$.

Finally, we assume that the innovations $\varepsilon_{r, t}, \varepsilon_{z, t}, \varepsilon_{q, t}, \varepsilon_{\pi^{*}, t}$, and $\varepsilon_{y^{*}, t}$ are independent standard normal random variables. We stack these innovations in the vector $\varepsilon_{t}$. 


\section{The DSGE-VAR Approach}

To capture potential misspecification of the stylized small open economy model described in the previous section, we embed it into a vector autoregressive specification that allows us to relax crosscoefficient restrictions. We refer to the resulting empirical model as DSGE-VAR. We have developed this DSGE-VAR framework in a series of papers, including Del Negro and Schorfheide (2004, 2008b) and Del Negro and others (2007). The remainder of this section reviews the setup in Del Negro and Schorfheide (2008b), which is used in the subsequent empirical analysis.

\subsection{A VAR with Hierarchical Prior}

Equation (2), which describes the policymaker's behavior, can be written in more general form as

$y_{1, t}=\mathbf{x}_{t}^{\prime} \boldsymbol{\beta}_{1}(\boldsymbol{\theta})+\mathbf{y}_{2, t}^{\prime} \boldsymbol{\beta}_{2}(\boldsymbol{\theta})+\varepsilon_{1, t} \sigma_{r}$,

where $\mathbf{y}_{t}=\left[y_{1, t}, \mathbf{y}_{2, t}^{\prime}\right]^{\prime}$ and the $k \times 1$ vector $\mathbf{x}_{t}=\left[\mathbf{y}_{t-1}^{\prime}, \ldots, \mathbf{y}_{t-p}^{\prime}, 1\right]^{\prime}$ is composed of the first $p$ lags of $\mathbf{y}_{t}$ and an intercept. Here $y_{1, t}$ corresponds to the nominal interest rate, $\tilde{R}_{t}$, while the subvector $\mathbf{y}_{2, t}$ is composed of output growth, inflation, exchange rate depreciation, and terms-of-trade changes:

$\mathbf{y}_{2, t}=\left[\left(\Delta \hat{y}_{t}+\hat{z}_{t}\right), \hat{\pi}_{t}, \hat{e}_{t}, \hat{q}_{t}\right]$.

The vector-valued functions $\boldsymbol{\beta}_{1}(\boldsymbol{\theta})$ and $\boldsymbol{\beta}_{2}(\boldsymbol{\theta})$ interact with $\mathbf{x}_{t}$ and $\mathbf{y}_{2, t}$ to reproduce the policy rule.

The solution of the linearized DSGE model presented in section 1 generates a moving-average representation of $\mathbf{y}_{2, t}$ in terms of $\varepsilon_{t}$. We proceed by approximating this moving-average representation with a $p$ th-order autoregression, which we write as

$\mathbf{y}_{2, t}=\mathbf{x}_{t}^{\prime} \Psi^{*}(\theta)+\mathbf{u}_{2, t}^{\prime}$

If we ignore the approximation error for a moment, the one-stepahead forecast errors, $\mathbf{u}_{2, t}$, are functions of structural innovations $\varepsilon_{t}$. 
Assuming that, under the DSGE model, the law of motion for $\mathbf{y}_{2, t}$ is covariance stationary for every $\theta$, we define the moment matrices

$\Gamma_{X X}(\theta)=E_{\theta}^{D}\left[\mathbf{x}_{t} \mathbf{x}_{t}^{\prime}\right]$

and

$\boldsymbol{\Gamma}_{X Y_{2}}(\boldsymbol{\theta})=E_{\theta}^{D}\left[\mathbf{x}_{t} \mathbf{y}_{2, t}^{\prime}\right]$

In our notation, $E_{\theta}^{D}[\cdot]$ denotes an expectation taken under the probability distribution for $\mathbf{y}_{t}$ and $\mathbf{x}_{t}$ generated by the DSGE model conditional on the parameter vector $\boldsymbol{\theta}$. We define the VAR approximation of $\mathbf{y}_{2, t}$ through

$\Psi^{*}(\theta)=\Gamma_{X X}^{-1}(\theta) \Gamma_{X Y_{2}}(\theta)$.

The equation for the policy instrument (12) can be rewritten by replacing $\mathbf{y}_{2, t}$ with expression (13):

$y_{1, t}=\mathbf{x}_{t}^{\prime} \boldsymbol{\beta}_{1}(\boldsymbol{\theta})+\mathbf{x}_{t}^{\prime} \Psi^{*}(\boldsymbol{\theta}) \boldsymbol{\beta}_{2}(\boldsymbol{\theta})+u_{1, t}$.

Let $\mathbf{u}_{t}^{\prime}=\left[u_{1, t}, \mathbf{u}_{2, t}^{\prime}\right]$ and define

$\Sigma^{*}(\theta)=\Gamma_{Y Y}(\theta)-\Gamma_{Y X}(\theta) \Gamma_{X X}^{-1}(\theta) \Gamma_{X Y}(\theta)$

If we assume that the $\mathbf{u}_{t}$ variables are normally distributed, denoted by $\mathbf{u}_{t} \sim \mathrm{N}\left(0, \boldsymbol{\Sigma}_{*}(\boldsymbol{\theta})\right)$, then equations (13) to (16) define a restricted $\operatorname{VAR}(p)$ for the vector $\mathbf{y}_{t}$. While the moving-average representation of $\mathbf{y}_{t}$ under the linearized DSGE model does not, in general, have an exact VAR representation, the restriction functions $\Psi^{*}(\theta)$ and $\Sigma^{*}(\theta)$ are defined such that the covariance matrix of $\mathbf{y}_{t}$ is preserved. Let $E_{\Psi, \Sigma}^{\mathrm{VAR}}[\cdot]$ denote expectations under the restricted VAR. It can be verified that

$E_{\Psi^{*}(\theta), \Sigma^{*}(\theta)}^{V A R}\left[\mathbf{y}_{t} \mathbf{y}_{t}^{\prime}\right]=E_{\theta}^{D}\left[\mathbf{y}_{t} \mathbf{y}_{t}^{\prime}\right]$.

To account for potential misspecification we now relax the DSGE model restrictions and allow for VAR coefficient matrices $\boldsymbol{\Psi}$ and $\boldsymbol{\Sigma}$ 
that deviate from the restriction functions $\Psi^{*}(\theta)$ and $\Sigma^{*}(\theta)$. Thus,

$y_{1, t}=\mathbf{x}_{t}^{\prime} \boldsymbol{\beta}_{1}(\boldsymbol{\theta})+\mathbf{x}_{t}^{\prime} \mathbf{\Psi} \boldsymbol{\beta}_{2}(\boldsymbol{\theta})+u_{1, t}$,

$\mathbf{y}_{2, t}=\mathbf{x}_{t}^{\prime} \mathbf{\Psi}+\mathbf{u}_{2, t}^{\prime}$,

and $\mathbf{u}_{t} \sim N(0, \boldsymbol{\Sigma})$. Our analysis is cast in a Bayesian framework in which initial beliefs about the DSGE-model parameter $\boldsymbol{\theta}$ and the VAR parameters $\boldsymbol{\Psi}$ and $\boldsymbol{\Sigma}$ are summarized in a prior distribution. Our prior distribution for $\boldsymbol{\Psi}$ and $\boldsymbol{\Sigma}$ is chosen such that, conditional on a DSGE-model parameter $\boldsymbol{\theta}$,

$\Sigma \mid \theta \sim I W\left(T^{*} \Sigma^{*}(\theta), T^{*}-k\right)$,

$\boldsymbol{\Psi} \mid \boldsymbol{\Sigma}, \boldsymbol{\theta} \sim N\left(\boldsymbol{\Psi}^{*}(\boldsymbol{\theta}), \frac{1}{T^{*}}\left[\left(\mathbf{B}_{2}(\boldsymbol{\theta}) \boldsymbol{\Sigma}^{-1} \mathbf{B}_{2}(\boldsymbol{\theta})^{\prime}\right) \otimes \boldsymbol{\Gamma}_{X X}(\boldsymbol{\theta})\right]^{-1}\right)$,

where $I W$ denotes the inverted Wishart distribution, $N$ is a multivariate normal distribution, $\mathbf{B}_{1}(\boldsymbol{\theta})=\left[\boldsymbol{\beta}_{1}(\boldsymbol{\theta}), \mathbf{0}_{k \times(n-1)}\right]$, and $\mathbf{B}_{2}(\boldsymbol{\theta})=\left[\boldsymbol{\beta}_{2}(\boldsymbol{\theta}), \mathbf{I}_{(n-1) \times(n-1)}\right]$.

Our hierarchical prior is computationally convenient. We use Markov-chain-Monte-Carlo (MCMC) methods (described in Del Negro and Schorfheide, 2008b) to generate draws from the joint posterior distribution of $\boldsymbol{\Psi}, \boldsymbol{\Sigma}$, and $\boldsymbol{\theta}$. We refer to the empirical model comprising the likelihood function associated with the restricted VAR in equation and the prior distributions $p_{\lambda}(\Psi, \Sigma \mid \theta)$, given in equation (18), and $p(\theta)$ as DSGE-VAR $(\lambda)$.

\subsection{Selecting the Tightness of the Prior}

The distribution of prior mass around the restriction functions $\Psi^{*}(\theta)$ and $\boldsymbol{\Sigma}^{*}(\boldsymbol{\theta})$ is controlled by the hyperparameter $T^{*}$, which we reparameterize in terms of multiples of the actual sample size $T$, that is, $T^{*}=\lambda T$. Large values of $\lambda$ imply that large discrepancies are unlikely to occur and the prior concentrates near the restriction functions. We consider values of $\lambda$ on a finite grid, $\Lambda$, and use a data-driven procedure to determine an appropriate value for this hyperparameter. A natural criterion to select $\lambda$ in a Bayesian framework is the marginal data density:

$p_{\lambda}(Y)=\int p(Y \mid \boldsymbol{\Psi}, \boldsymbol{\Sigma}, \boldsymbol{\theta}) p_{\lambda}(\boldsymbol{\Psi}, \boldsymbol{\Sigma}, \boldsymbol{\theta}) d(\boldsymbol{\Psi}, \boldsymbol{\Sigma}, \boldsymbol{\theta})$. 
Here $p_{\lambda}(\boldsymbol{\Psi}, \boldsymbol{\Sigma}, \boldsymbol{\theta})$ is a joint prior distribution for the VAR coefficient matrices and the DSGE model parameters. This prior is obtained by combining the prior in equation (18) with a prior density for $\boldsymbol{\theta}$, denoted by $p(\theta)$ :

$p_{\lambda}(\boldsymbol{\Psi}, \boldsymbol{\Sigma}, \boldsymbol{\theta})=p(\boldsymbol{\theta}) p_{\lambda}(\boldsymbol{\Sigma} \mid \boldsymbol{\theta}) p_{\lambda}(\boldsymbol{\Phi} \mid \boldsymbol{\Sigma}, \boldsymbol{\theta})$.

Suppose that $\Lambda$ consists of only two values, $\lambda_{1}$ and $\lambda_{2}$. Moreover, suppose that the econometrician places equal prior probability on these two values. The posterior odds of $\lambda_{1}$ versus $\lambda_{2}$ are given by the marginal likelihood ratio, $p_{\lambda_{1}}(Y) / p_{\lambda_{2}}(Y)$. More generally, if the grid consists of $J$ values that have equal prior probability, then the posterior probability of $\lambda_{j}$ is proportional to $p_{\lambda_{j}}(Y), j=1, \ldots, J$. Rather than averaging our conclusions with respect to the posterior distribution of the lambdas, we condition on the value $\lambda_{j}$ that has the highest posterior probability. Such an approach is often called empirical Bayes analysis in the literature. In particular, we define

$$
\hat{\lambda}=\operatorname{argmax}_{\lambda \in \Lambda} p_{\lambda}(Y) .
$$

As discussed in Del Negro and others (2007), the marginal likelihood ratio, $p_{\lambda=\hat{\chi}}(Y) / p_{\lambda=\infty}(Y)$, provides an overall measure of fit for the DSGE model. If the data are not at odds with the restrictions implied by the DSGE model-that is, there exists a parameterization, $\tilde{\boldsymbol{\theta}}$, of the DSGE model for which the model-implied autocovariances are similar to the sample autocovariances-then $\hat{\lambda}$ will be large and $p_{\lambda=\hat{\lambda}}(Y) / p_{\lambda=\infty}(Y)$ will be small. If the data turn out to be at odds with the DSGE model implications, $\hat{\lambda}$ will be fairly small and $p_{\lambda=\hat{\lambda}}(Y) / p_{\lambda=\infty}(Y)$ will be large. We come back to the interpretation of these marginal likelihood ratios when we discuss the empirical results.

\subsection{Identification of Structural Shocks}

Up to this point, we have expressed the VAR in terms of one-stepahead forecast errors, $\mathbf{u}_{t}$. It is more useful, however, to express the VAR as a function of the structural shocks, $\varepsilon_{t}$, both for understanding the dynamics of the DSGE-VAR and for the purpose of policy analysis. 
In our setup, the monetary policy shock is identified through exclusion restrictions:

$y_{1, t}=\mathbf{x}_{t}^{\prime} \boldsymbol{\beta}_{1}(\boldsymbol{\theta})+\left(\mathbf{x}_{t}^{\prime} \boldsymbol{\Psi}+\mathbf{u}_{2, t}^{\prime}\right) \boldsymbol{\beta}_{2}(\boldsymbol{\theta})+\varepsilon_{1, t} \sigma_{R} ;$

$\mathbf{y}_{2, t}^{\prime}=\mathbf{x}_{t}^{\prime} \Psi+\mathbf{u}_{2, t}^{\prime}$.

According to the underlying DSGE model, $\mathbf{u}_{2, t}$ is a function of the monetary policy shock, $\varepsilon_{1, t}$, and other structural shocks, $\varepsilon_{2, t}$. We assume that the shocks $\varepsilon_{2, t}$ have unit variance and are uncorrelated with each other or with the monetary policy shock. We express $\mathbf{u}_{2, t}$ as

$\mathbf{u}_{2, t}^{\prime}=\varepsilon_{1, t} \mathbf{A}_{1}+\varepsilon_{2, t}^{\prime} \mathbf{A}_{2}$

Straightforward matrix algebra leads to the following formulas for the effect of the structural shocks on $\mathbf{u}_{2, t}^{\prime}$ :

$$
\begin{aligned}
& \mathbf{A}_{1}=\left[\boldsymbol{\Sigma}_{11}-\boldsymbol{\beta}_{2}^{\prime} \boldsymbol{\Sigma}_{22} \boldsymbol{\beta}_{2}-2\left(\boldsymbol{\Sigma}_{12}-\boldsymbol{\beta}_{2}^{\prime} \boldsymbol{\Sigma}_{22}\right) \boldsymbol{\beta}_{2}\right]^{-1}\left(\boldsymbol{\Sigma}_{12}-\boldsymbol{\beta}_{2}^{\prime} \boldsymbol{\Sigma}_{22}\right) \\
& \mathbf{A}_{2}^{\prime} \mathbf{A}_{2}=\boldsymbol{\Sigma}_{22}-\mathbf{A}_{1}^{\prime}\left[\boldsymbol{\Sigma}_{11}-\boldsymbol{\beta}_{2}^{\prime} \boldsymbol{\Sigma}_{22} \boldsymbol{\beta}_{2}-2\left(\boldsymbol{\Sigma}_{12}-\boldsymbol{\beta}_{2}^{\prime} \boldsymbol{\Sigma}_{22}\right) \boldsymbol{\beta}_{2}\right] \mathbf{A}_{1} .
\end{aligned}
$$

While the above decomposition of the forecast error covariance matrix identifies $\mathbf{A}_{1}$, it does not uniquely determine the matrix $\mathbf{A}_{2}$. To do so, we follow the approach taken in Del Negro and Schorfheide (2004). Let $\mathbf{A}_{2, t r}^{\prime} \mathbf{A}_{2, t r}=\mathbf{A}_{2}^{\prime} \mathbf{A}_{2}$ be the Cholesky decomposition of $\mathbf{A}_{2}^{\prime} \mathbf{A}_{2}$. The relationship between $\mathbf{A}_{2, t r}$ and $\mathbf{A}_{2}$ is given by $\mathbf{A}_{2}^{\prime}=\mathbf{A}_{2, t r}^{\prime} \boldsymbol{\Omega}$, where $\boldsymbol{\Omega}$ is an orthonormal matrix that is not identifiable based on the estimates of $\boldsymbol{\beta}(\boldsymbol{\theta}), \boldsymbol{\Psi}$, and $\boldsymbol{\Sigma}$. However, we are able to calculate an initial effect of $\varepsilon_{2, t}$ on $\mathbf{y}_{2, t}$ based on the DSGE model, denoted by $\mathbf{A}_{2}^{D}(\boldsymbol{\theta})$. This matrix can be uniquely decomposed into a lower triangular matrix and an orthonormal matrix:

$\mathbf{A}_{2}^{D^{\prime}}(\boldsymbol{\theta})=\mathbf{A}_{2, t r}^{D^{\prime}}(\boldsymbol{\theta}) \boldsymbol{\Omega}^{*}(\boldsymbol{\theta})$

To identify $\mathbf{A}_{2}$ above, we combine $\mathbf{A}_{2, t r}^{\prime}$ with $\boldsymbol{\Omega}^{*}(\boldsymbol{\theta})$. Loosely speaking, the rotation matrix is constructed such that in the absence of misspecification, the DSGE model's and the DSGE-VAR's impulse responses to $\varepsilon_{2, t}$ coincide. To the extent that misspecification is mainly in the dynamics as opposed to the covariance matrix of innovations, 
the identification procedure can be interpreted as matching, at least qualitatively, the short-run responses of the VAR with those from the DSGE model. Since the matrix $\Omega$ does not affect the likelihood function, we can express the joint distribution of data and parameters as follows:

$p_{\lambda}(Y, \Psi, \boldsymbol{\Sigma}, \boldsymbol{\Omega}, \boldsymbol{\theta})=p(Y \mid \boldsymbol{\Psi}, \boldsymbol{\Sigma}) p_{\lambda}(\boldsymbol{\Psi}, \boldsymbol{\Sigma} \mid \boldsymbol{\theta}) p(\boldsymbol{\Omega} \mid \boldsymbol{\theta}) p(\boldsymbol{\theta})$.

Here $p(\boldsymbol{\Omega} \mid \boldsymbol{\theta})$ is a point mass centered at $\boldsymbol{\Omega}^{*}(\boldsymbol{\theta})$. The presence of $\boldsymbol{\Omega}$ does not affect the MCMC algorithm. We can first draw the triplet $\boldsymbol{\Psi}, \boldsymbol{\Sigma}$, $\boldsymbol{\theta}$ from the posterior distribution associated with the reduced-form DSGE-VAR, and then calculate $\boldsymbol{\Omega}$ according to $\boldsymbol{\Omega}^{*}(\boldsymbol{\theta})$. Details are provided in Del Negro and Schorfheide (2008b).

\section{Data}

For our empirical analysis, we compiled a data set of observations on output growth, inflation, interest rates, exchange rates, and the terms of trade. Unless otherwise noted, the raw data are taken from the online database maintained by the Central Bank of Chile and seasonally adjusted. Output growth is defined as the log difference of real gross domestic product (GDP), scaled by 400 to convert it into annualized percentages. To construct the inflation series, we pass the consumer price index extracted from the Central Bank database through the X12 filter (using the default settings in EViews) to obtain a seasonally adjusted series; we then compute log differences, scaled by 400 . The monetary policy rate (MPR) serves as our measure of nominal interest rates. ${ }^{3}$ Annualized depreciation rates are computed from log differences of the Chilean peso / U.S. dollar exchange rate series. Finally, annualized quarter-to-quarter percentage changes in the terms of trade are computed from the export and import price indexes maintained by the Central Bank.

While we compile a data set that contains quarterly observations from 1986 to 2007 , we restrict the estimation sample to the the period from 1999:1 to 2006:4 and hence to the most recent monetary policy regime. Between 1991 and 1999, the Central Bank applied a partial inflation-targeting approach that involved two nominal anchors: an exchange rate band and an inflation target. In 1999

3. To construct the MPR before 2001, we follow the approach in Chumacero (2005). 
the Central Bank implemented a floating exchange rate and the institutional arrangements for full inflation targeting. ${ }^{4}$ Official bank publications state that the operating objective of monetary policy is to keep annual inflation projections around 3.0 percent annually over a horizon of about two years. Indeed, the average inflation rate in our estimation sample is 2.8 percent. We plot the path of the inflation rate and the nominal interest rate in figure 1 for the period 1986 to 2007. Chile experienced a decade-long disinflation process throughout the 1990s, and with the adoption of the 3 percent target inflation rate in 1999, inflation and nominal interest rates stabilized at a low level.

\section{Figure 1. Interest Rates and Inflation in Chile}

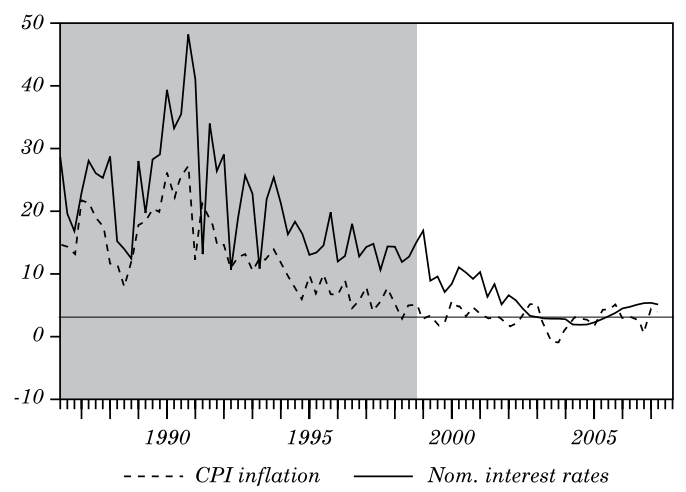

Sources: Central Bank of Chile and authors' calculations.

The average growth rate of real output (4.4 percent during our sample period) provides an estimate of $\gamma$ in equation (1). The average inflation rate can be viewed as an estimate of the target inflation rate $\pi_{*}$, and the average nominal interest rate can be linked to the discount factor $\beta$, because our model implies that $R_{*}=\gamma / \beta+\pi_{*}$. It turns out that the sum of average inflation and output growth is 7.2 percent and exceeds the average nominal interest rate, which is about 5.6 percent. The sample averages are thus inconsistent with the model's steady-state implications. Rather than estimating the steady-state

4. Since 2000, the Central Bank of Chile has provided an inflation report with public inflation and growth forecasts. The inflation target has been stable at 3 percent since 2001. 
parameters jointly with the remaining DSGE model parameters and imposing the steady-state restrictions, we decided to demean our observations and fit the DSGE model and the DSGE-VAR to demeaned data.

To provide further details on the features of our data set, we plot the peso-dollar exchange rate in figure 2 , together with percentage changes in the terms of trade. Both series exhibit very little autocorrelation and are very volatile. According to our DSGE model, the exchange rate fluctuations are a function of inflation differentials and terms-of-trade movements:

$\hat{e}_{t}=\hat{\pi}_{t}-\hat{\pi}_{t}^{*}-(1-\alpha) \hat{q}_{t}$

The rest of the world's inflation rate, $\hat{\pi}_{t}^{*}$, is treated as a latent variable. In figure 3 we plot the exchange rate depreciation and the implicit inflation in the rest of the world, $-\hat{\pi}_{t}^{*}=\hat{e}_{t}-\hat{\pi}_{t}+(1-\alpha) \hat{q}_{t}$ for $\alpha=0.3$. The figure illustrates the well-known exchange rate disconnect: most of the fluctuations in the nominal exchange rate are generated by the exogenous process $\hat{\pi}_{t}^{*}$.

Figure 2. Exchange Rate and Terms of Trade Dynamics

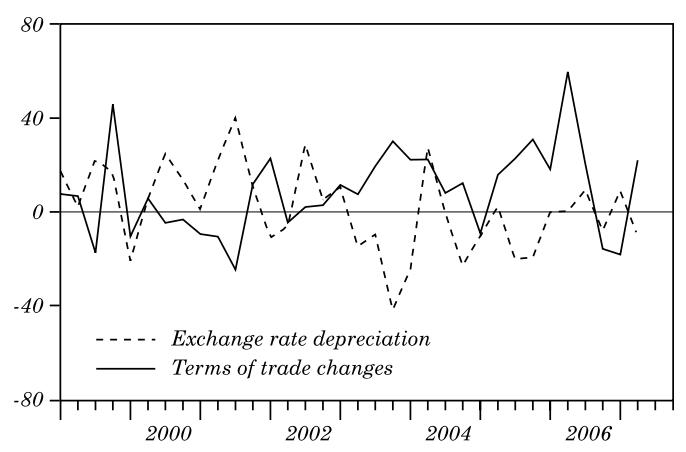

Sources: Central Bank of Chile and authors' calculations. 
Figure 3. Exchange Rate Movements and PPP

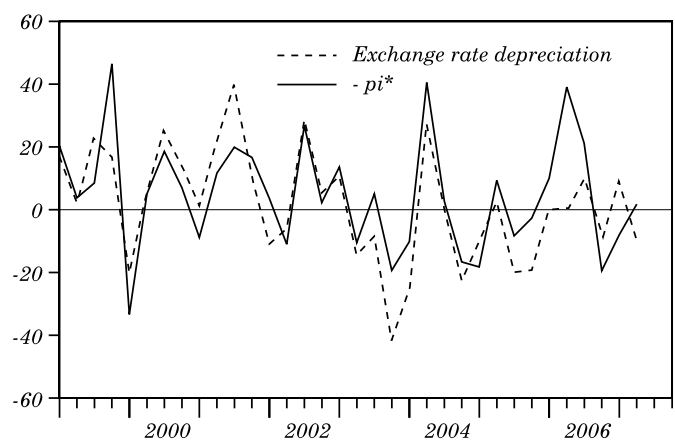

Sources: Central Bank of Chile and authors' calculations.

\section{Empirical Results}

The empirical analysis has four parts. In section 4.1 we estimate a monetary policy rule for Chile and examine the extent to which the Central Bank responds to exchange rate and terms-of-trade movements. We proceed in section 4.2 by studying the degree of misspecification of the DSGE model. More specifically, we compare the marginal likelihood of the DSGE model with that of the DSGE-VAR for various choices of the hyperparameter $\lambda$. Section 4.3 examines whether the Central Bank managed to insulate the Chilean economy, in particular inflation, from external shocks. Finally, section 4.4 explores the effect of changing the response to inflation in the feedback rule on the variance of inflation.

\subsection{Estimating the Policy Rule}

This section investigates the feedback rule followed by the Central Bank in the recent period. As discussed before, Chile witnessed significant movements in the nominal exchange rate after it entered the freely floating regime in 1999. Moreover, it was subject to large swings in the terms of trade. Did the Central Bank respond to these movements in order to pursue the inflation target? Table 1 addresses this question. The table estimates the coefficients of the policy rule (equation 2) under three different specifications. Under the first specification, which we refer to as the 
baseline, policy only responds to inflation and real output growth, in addition to the lagged interest rate. Under the second and third specifications (response to the exchange rate and response to the terms of trade, respectively), policy also responds to the exchange rate depreciation. Finally, the terms of trade also enter the feedback rule in the third specification, as do real output growth, inflation, and the nominal exchange rate. The posterior means of the policy rule coefficients estimated using the DSGE model under the three specifications are reported in columns (1a), (2a), and (3a) of the table, with the associated posterior standard deviations in parenthesis. For each specification we also compute the marginal likelihood, which measures model fit in a Bayesian framework, as well as the posterior odds relative to the baseline specification. This latter figure is computed under the assumption that we assign equal prior weights to all specifications.

Both posterior estimates of the parameters and model comparison results are, in a finite sample, sensitive to the choice of prior. ${ }^{5}$ Since the sample considered here is fairly short, we want to examine the robustness of our conclusions to the choice of prior. Table 1 thus presents our results for two priors, which differ in terms of the marginal distribution for two key parameters of interest: the policy responses to fluctuations in the exchange rate $\left(\psi_{3}\right)$ and in the terms of trade $\left(\psi_{4}\right)$. For the first prior, the marginal distribution for $\psi_{3}$ is centered at 0.25 with a standard deviation of 0.12 . This prior embodies the belief that the response to the exchange rate depreciation is on average substantial, but also quite diffuse. That is, it allows for the possibility that the response can either be small or very large. Likewise, the first prior is agnostic as to the response to the terms-of-trade depreciation. The prior is symmetric around zero, as we do not have a priori views on the sign of the response, and the standard deviation is quite large (0.50). This prior therefore allows for large positive or negative responses. The second is far less agnostic. Here the marginal distribution for $\psi_{3}$ is centered at 0.10 with a standard deviation of 0.05 . This prior embodies a relatively sharp belief that policy responds to depreciation, albeit not too strongly. The center of the marginal distribution on $\psi_{4}$ is still zero, but the standard deviation is 0.10 , five times smaller than in the case of the first prior.

5. See Del Negro and Schorfheide (2008a) for a discussion of prior elicitation and robustness in the context of DSGE models. 


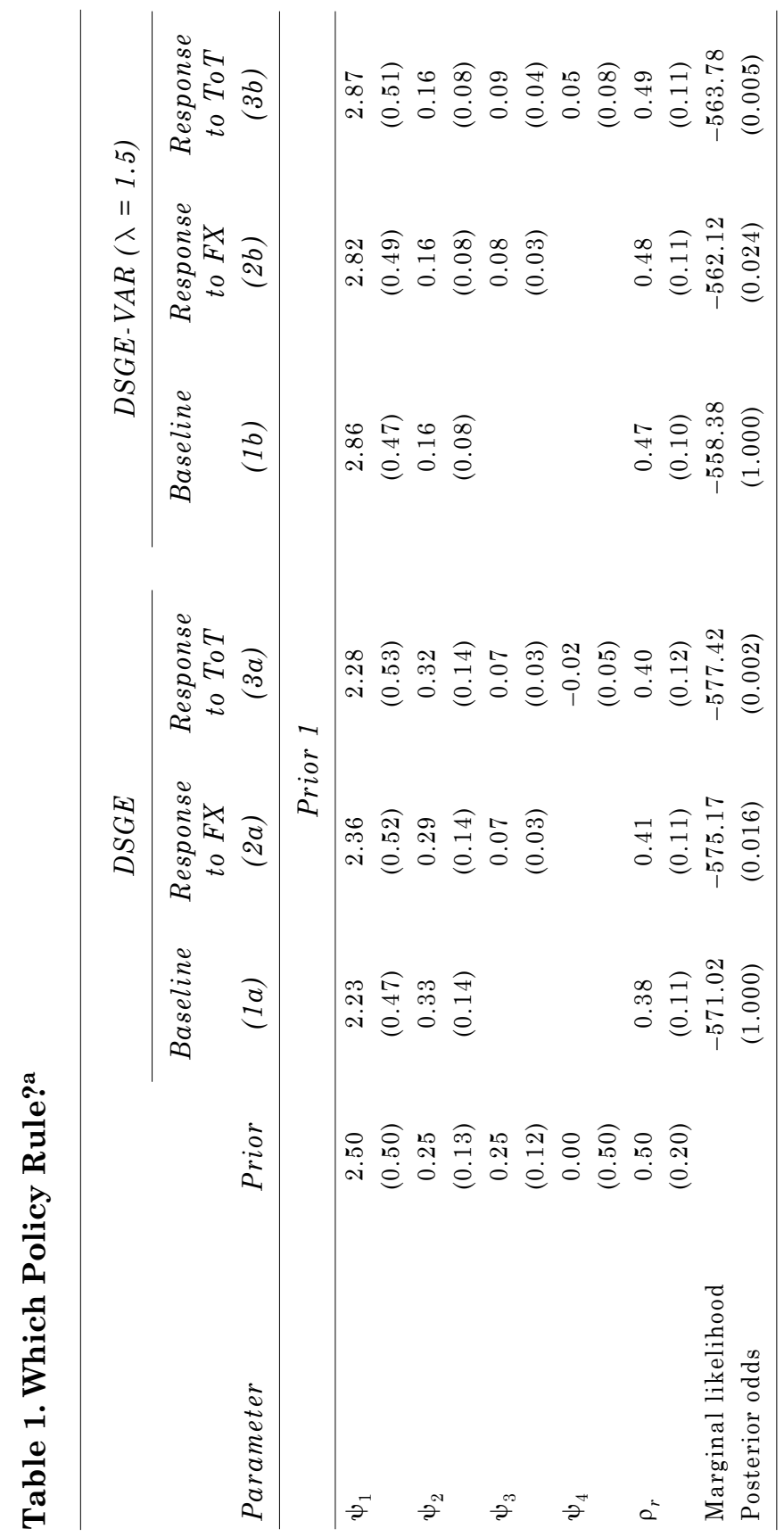




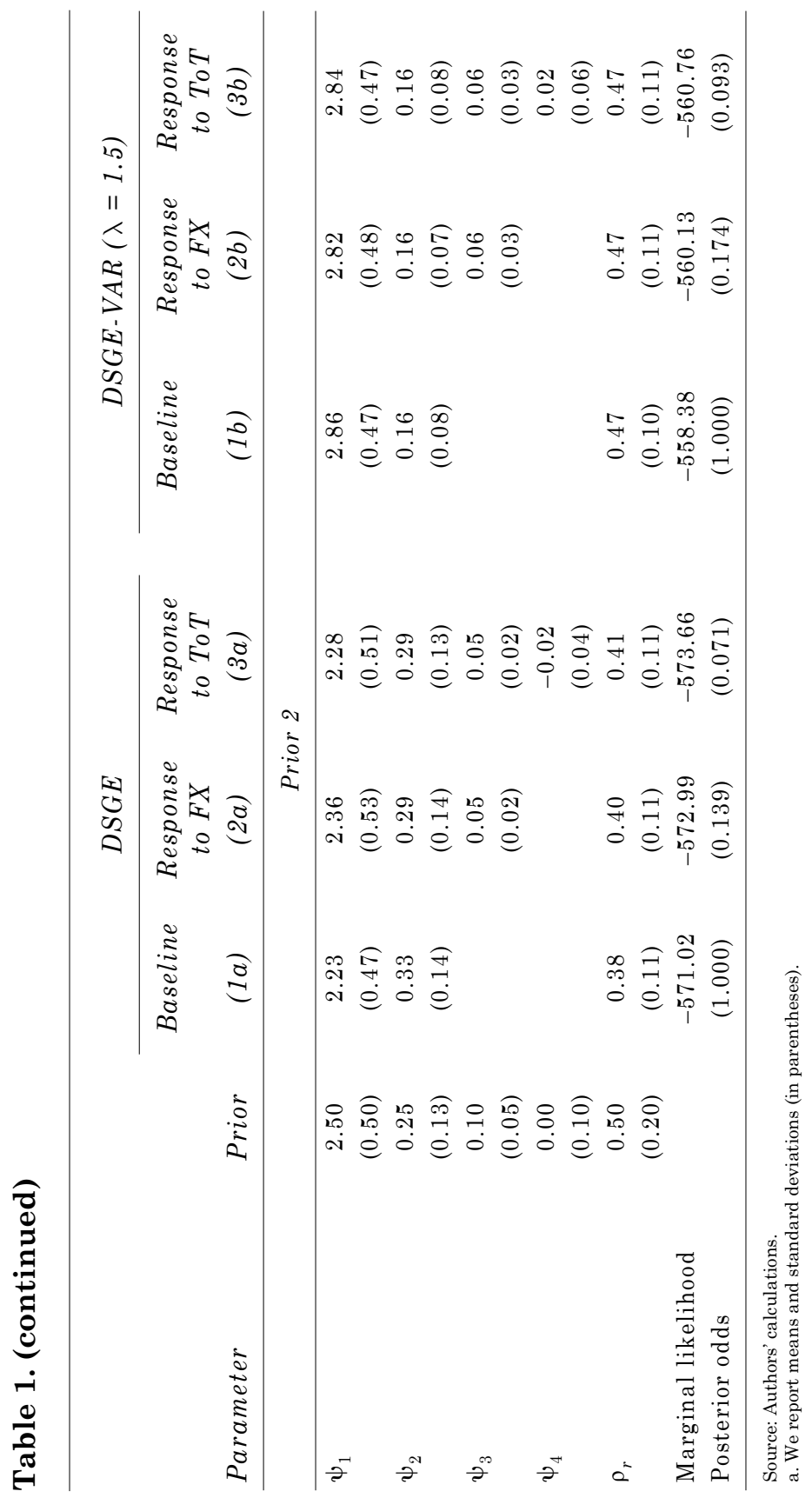


The distributions for the remaining policy parameters, $\psi_{1}, \psi_{2}$, and $\rho_{r}$, are the same across the two priors. Since Chile entered the full-fledged inflation-targeting regime in 1999 and it had acquired a reputation as an inflation fighter in the previous decade, we posit a fairly large prior mean on $\psi_{1}$, the response to inflation. The prior is centered at 2.50 with a standard deviation of 0.50 . The priors on $\psi_{2}$, the response to real output growth, and $\rho_{r}$, the persistence parameter, are similar to those used in Lubik and Schorfheide (2006). These priors are also similar to that used in the estimation of DSGE models for the United States. The prior distribution on the remaining DSGE model parameters are again the same across the two priors, and they are discussed in detail in the next section.

We now discuss the posterior estimates of the policy parameters for the three specifications, which are shown in columns (1a), (2a), and (3a) of table 1 . The estimates of $\psi_{1}$ are consistent across specifications, ranging from 2.23 to 2.36 , with a standard deviation of about 0.5 . Our prior was that the Central Bank responds strongly to inflation, and there is little updating from the prior to the posterior. ${ }^{6}$ The estimates of $\psi_{2}$ range from 0.29 to 0.33 and imply only modest updating relative to the prior. The estimates for $\psi_{1}$ and $\psi_{2}$ are also roughly the same for both priors.

The main focus of the section lies in the responses to nominal depreciation and to the terms of trade. In these dimensions the data are quite informative. Column (2a) reveals that under the first prior, the posterior mean for $\psi_{3}$, the response to nominal depreciation, is 0.07 , much lower than the prior mean. Moreover, the posterior is much more concentrated than the prior. The data strongly indicate that the response to exchange rate depreciations, if at all nonzero, is much smaller than the response to CPI inflation. To put this estimate into perspective, define the target nominal interest rate as

$$
\hat{R}_{t}^{*}=2.36 \hat{\pi}_{t}+0.29\left(\Delta \hat{y}_{t}+\hat{z}_{t}\right)+0.07 \hat{e}_{t} .
$$

6. Since the official inflation target is stated in terms of year-over-year inflation, we also consider a fourth specification in which we replace quarter-to-quarter inflation. We find that this specification is strongly rejected by the data using our posterior odds criterion. This result should not be interpreted as contradicting the statement that the Central Bank target is year-over-year inflation, but simply as providing information on the rule the Central Bank follows to achieve this target. Caputo and Liendo (2005) consider a rule in which the policymaker responds to expected inflation and find it does not improve fit. 
Here we replaced the policy rule coefficients by their posterior mean estimates. The sample standard deviations of inflation, output growth, and nominal exchange rate depreciations are 1.57, 3.77, and 18.10, respectively. We can therefore rewrite the target interest rate as

$$
\hat{R}_{t}^{*}=3.17 \frac{\hat{\pi}_{t}}{\hat{\sigma}(\hat{\pi})}+1.09 \frac{\Delta \hat{y}_{t}+\hat{z}_{t}}{\hat{\sigma}(\Delta \hat{y}+\hat{z})}+1.27 \frac{\hat{e}_{t}}{\hat{\sigma}(\hat{e})} .
$$

After the standardization, the coefficient on the exchange rate depreciation is 1.27 , whereas the coefficient on CPI inflation is 3.17.

Column (3a) shows that when we further add the response to the terms of trade to the feedback rule, the estimated coefficient $\boldsymbol{\psi}_{4}$ under the first prior is negative but small. Our standardized posterior mean estimate for the terms-of-trade coefficient is -0.37 . The posterior standard deviation is also relatively small, indicating that the data rule out a large response. The marginal likelihood and posterior odds show that under the first prior, the alternative specifications are rejected by the data. The posterior odds relative to the baseline are 1.6 and 0.2 percent, respectively.

It is conceivable that the response to the exchange rate or terms of trade, while not as important as that of inflation, is still significant. We embody this belief in the second prior. The posterior mean of $\psi_{3}$ under the second prior is 0.05 , which is smaller than under the first prior. However, $\psi_{3}$ is now more precisely estimated. The posterior mean of $\psi_{4}$ is the same under both priors, while the posterior standard deviation decreases by 0.01 under the second prior. Even under the tighter prior, the posterior odds favor the baseline specification. To summarize, based on the DSGE model estimation, we conclude that responding to inflation is much more important for the Central Bank than responding to the exchange rate or the terms of trade.

Full-information estimation has pros and cons if one is interested in the parameters of a particular equation in the system, in this case the policy rule. On the one hand, if the cross-equation restrictions imposed by the model are correct, full-information estimation is more efficient than single-equation instrumental variable estimation. On the other hand, to the extent that these cross-equation restrictions are invalid, the full-information estimates are potentially biased, and limited-information methods may be preferable. In this context, DSGE-VAR strikes a compromise between full- and limited-information estimation, as it allows for deviations from the 
cross-equation restrictions. In the case at hand, such a compromise may be necessary, since the sample size is small and estimators that completely ignore the restrictions $(\lambda=0)$ tend to produce poor estimates in a mean-squared-error sense. At the same time, our DSGE model generates strong cross-equation restrictions (such as exogeneity of the terms of trade), so we may not want to impose them dogmatically $(\lambda=\infty)$. For these reasons, columns (1b), (2b), and (3b) of table 1 show the estimates of $\psi_{1}, \psi_{2}, \psi_{3}$, and $\rho_{r}$ according to the three specifications of interest using a DSGE-VAR with two lags and $\lambda=1.5$. We justify the choice of lag length and hyperparameter in section 4.2. For now, notice that the marginal likelihood of the DSGE-VAR for all specifications is substantially higher than that of the corresponding DSGE model, validating some of the concerns about the cross-equation restrictions.

The DSGE-VAR estimates imply a stronger response to inflation and a weaker response to output growth than the DSGE model estimates, with a posterior mean of $\psi_{1}$ between 2.8 and 2.9 and $\psi_{2}$ at 0.16 . The DSGE-VAR estimation confirms our previous findings regarding the response to exchange rate and terms-of-trade movements. Under the first prior the posterior means of $\psi_{3}$ are 0.08 and 0.09 for the second and third specifications, respectively. The posterior mean for $\psi_{4}$, the response to terms-of-trade changes, has the opposite sign than under the DSGE estimation, but it is still relatively small. Most importantly, the posterior odds suggest that the richer specifications are rejected relative to the baseline. Under the second prior, the estimates for $\psi_{3}$ are also in line with those obtained under the DSGE estimation. The estimates for $\psi_{4}$ again have the opposite sign, but are close to zero.

As emphasized by Galí and Monacelli (2005), optimal monetary policy in our DSGE model would consist of stabilizing domestic inflation, $\hat{\pi}_{H, t}$, and the gap between actual and flexible price output, $\hat{y}_{t}-\bar{y}_{t}$. Since according to our model $\hat{\pi}_{H, t}=\hat{\pi}_{t}+\alpha \hat{q}_{t}$, and the estimated import share $\alpha$ is between 25 and 30 percent, our posterior estimates in columns (3a) and (3b) of table 1 suggest that the Central Bank does not try to stabilize $\hat{\pi}_{H, t}$.

In summary, we have robust empirical evidence that the Central Bank responded only very mildly to movements in the nominal exchange rate or the terms of trade in the recent period, if it responded at all. Rather, CPI inflation is the driving force behind changes in interest rates. Our post-1999 findings are consistent with the official policy statements of the Central Bank of Chile. 


\subsection{The Fit of the Small Open Economy DSGE Model}

This section discusses the fit of the small open economy DSGE model and the estimates of the nonpolicy parameters. More specifically, we examine how the fit of the DSGE-VAR changes as we relax the cross-equation restrictions implied by the DSGE model. From a policy perspective, this analysis is useful for assessing whether forecasting should be conducted with a tightly parameterized empirical specification that closely resembles the DSGE model, or with a densely parameterized VAR that uses little a priori information.

Table 2 shows the log marginal likelihood for the DSGE model and the DSGE-VAR, where $\lambda$ varies in a grid from 0.75 to 5.0. As discussed in section 2 , high values of $\lambda$ correspond to tightly imposed crossequation restrictions, while low values imply a relatively flat prior on the VAR parameters. The table also shows the posterior odds relative to the best-fitting model, which are computed under the assumption that all specifications have equal prior probabilities.

In previous studies that employ the DSGE-VAR methodology (Del Negro and Schorfheide, 2004; Del Negro and others, 2007), we use a VAR specification with four lags, which we denote VAR(4). Four lags are fairly standard in applications with twenty to forty years of quarterly data. Since we have fewer than nine years of data in the present application, an unrestricted estimation of a $\operatorname{VAR}(4)$ would imply that we are using only thirty-four observations to determine twenty parameters per equation. Consequently, a DSGE-VAR with four lags would require high values of $\lambda$, not because the DSGE model is a particularly good description of the data, but because only a very tight prior is able to reduce the variability of the estimates. We proceed by reducing the number of lags in the VAR. Columns (1) and (2) and columns (3) and (4) of the table show the log marginal likelihood and posterior odds results for DSGE-VARs with two and three lags, respectively.

Four features emerge from table 2. First, for any value of $\lambda$, the log marginal likelihood for two lags (column 1) is always greater than that for three lags (column 3), indicating that reducing the number of lags, and hence the number of free parameters, increases the fit of the empirical model. If we raise the number of lags to four, the log marginal likelihood decreases even further. Second, the gap in marginal likelihoods between columns (1) and (3) tends to decrease with $\lambda$ : increasing the weight of the DSGE model's restrictions implicitly decreases the number of free parameters and hence makes the difference between VARs with two and three lags less stark. 


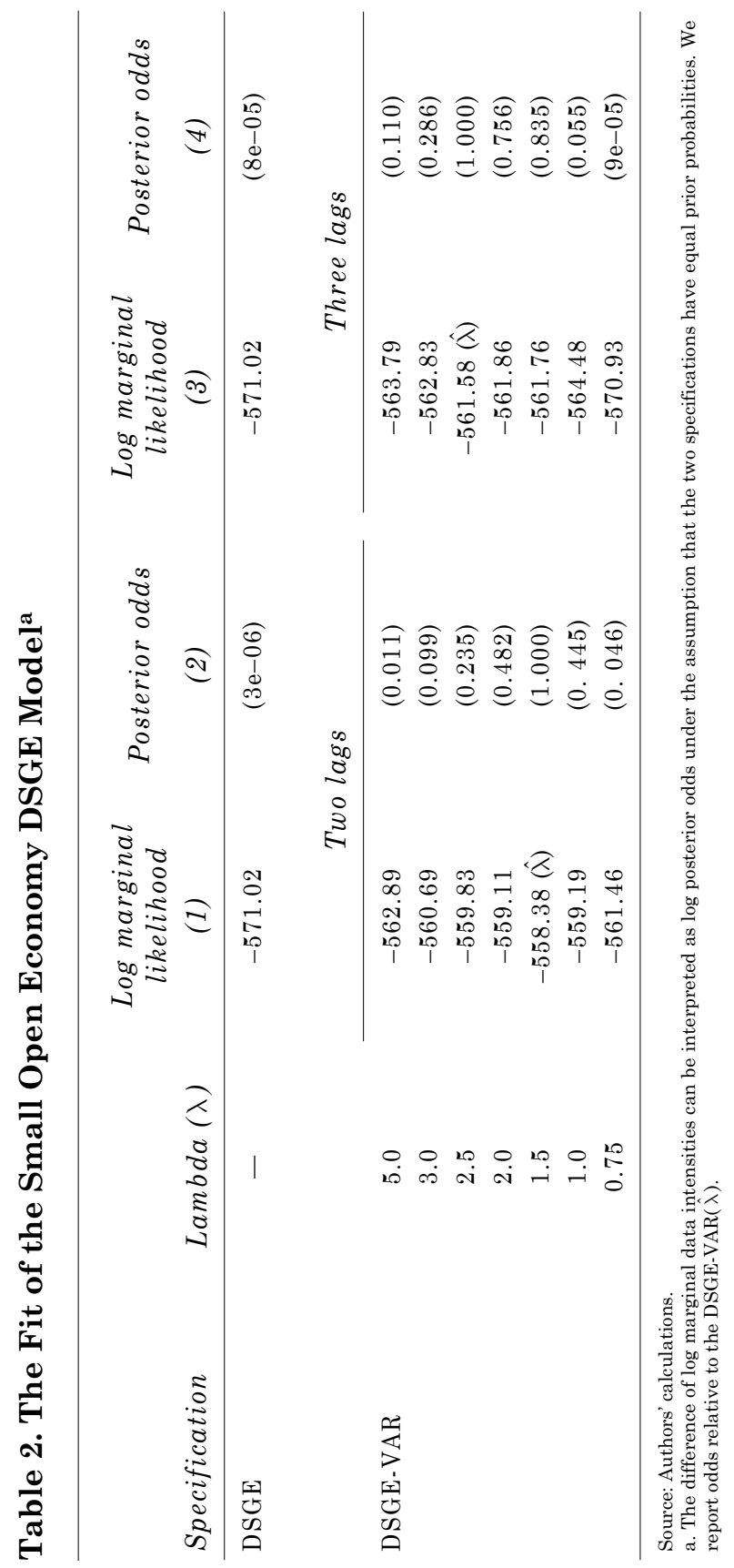


Third, the best fit is achieved for a value of $\lambda$ that is lower for the $\operatorname{VAR}(2)$ than the VAR(3) specification. Using the notation of section $2, \hat{\lambda}$ takes the values 1.5 and 2.5 , respectively. The DSGE model restrictions help in part because they reduce the number of free parameters, and this reduction becomes more valuable the larger the lag length. ${ }^{7}$ Finally, the fit of the DSGE model is considerably worse than that of the DSGE-VAR $(\hat{\lambda})$, regardless of the number of lags. Columns (2) and (4) of table 2 show that the posterior odds of the DSGE model relative to DSGE-VAR $(\hat{\lambda})$ are 1 percent and 10 percent, respectively, indicating that from a statistical point of view there is evidence that the cross-equation restrictions are violated in the data. We investigate in section 4.3 whether this statistical evidence is economically important, that is, whether it translates into sizeable differences with respect to the dynamic response of the endogenous variables to different shocks.

Table 3 provides the estimates of the DSGE model's nonpolicy parameters. We focus on the estimates obtained with the the two-lag $\operatorname{DSGE}-\operatorname{VAR}(\hat{\lambda})$. Results for the $\operatorname{VAR}(3)$ are quantitatively similar. The first column of the table shows the prior mean and standard deviations. The parameter $\alpha$ measures the fraction of foreign-produced goods in the domestic consumption basket. In 2006 imported goods as a share of total domestic demand in Chile was about 30 percent. Restricted to consumer goods, this share was 10 percent. We decided to center our prior at the 30 percent value, allowing for substantial variation. The parameter $r^{*}$ can be interpreted as the growth-adjusted real interest rate. While our observations on average GDP growth, inflation, and nominal interest rates between 1999 and 2007 suggest that this value is negative, we view this as a temporary phenomenon and center our prior for $r^{*}$ at 2.5 percent. The parameter $\kappa$ corresponds to the slope of the Phillips curve, which captures the degree of price stickiness. According to our prior, $\kappa$ falls with high probability in the interval 0 to 1 , which encompasses large nominal rigidities as well as the case of near flexible prices. The parameter $\tau$ captures the inverse of the relative risk aversion. We center our prior at 2 , which implies that consumers are slightly more risk averse than consumers with a log utility function. Finally, the priors for the parameters of the exogenous processes were chosen with presample evidence in mind.

7. Using the dummy observation interpretation of Del Negro and Schorfheide (2004), $\lambda=1.5$ implies that the actual data are augmented by $1.5 \times T$ artificial observations from the DSGE model. 


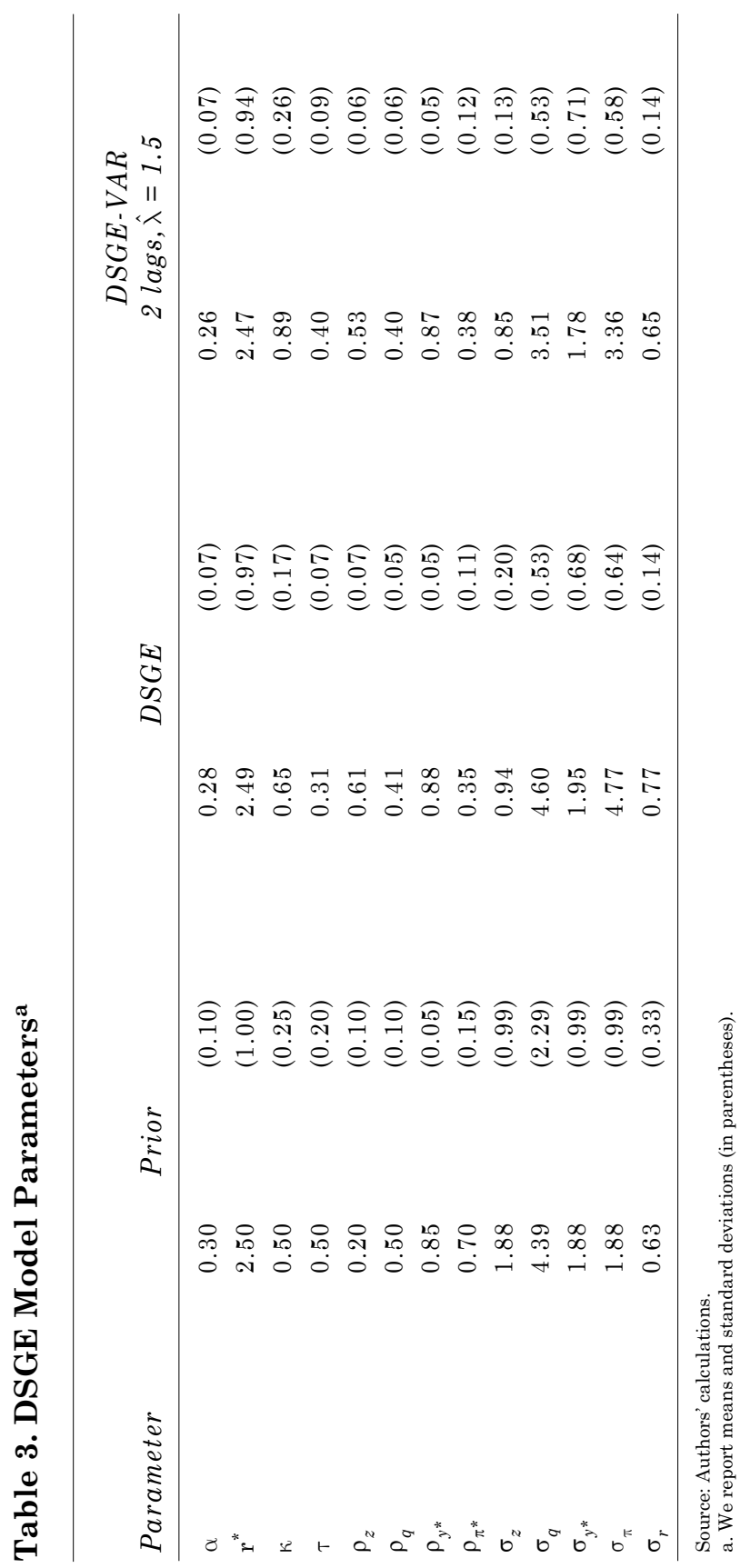


The second column shows the posterior mean and standard deviations obtained from the estimation of the DSGE model. In light of the DSGE model misspecification discussed above, it is important to ask whether accounting for deviations from the cross-equation restrictions affects the inference about the DSGE parameters. Therefore, the third column shows the estimates obtained using DSGE-VAR $(\hat{\lambda})$. The data provide little information on $r^{*}$, which enters the log-linear equations through the discount factor $\beta$, and the slope of the Phillips curve, $\kappa$. The estimated import share is about 25 percent, which again is not very different from the prior. The information from output, inflation, interest rate, exchange rate, and terms-of-trade data is not in contrast with that obtained from import quantities. Finally, the posterior mean of $\tau$ decreases compared to its prior, and its standard deviation shrinks from 0.2 to 0.1 or less. The posterior estimates for $\alpha, \kappa$, and $\tau$ are similar to those obtained by Caputo and Liendo (2005) for the 1999-2005 sample. The estimated standard deviation of the monetary policy shock is around 60 to 80 basis points. Overall, the parameter estimates obtained from the state-space representation of the DSGE model and the DSGE-VAR are very similar.

Since the DSGE model itself exhibits very little endogenous propagation, the dynamics of the data are mostly captured by the estimated autocorrelation parameters of the exogenous shock processes. The terms of trade are purely exogenous in the DSGE model, and thus the posterior means of $\rho_{q}$ and $\sigma_{q}$ measure the autocorrelation and innovation standard deviation in our terms-of-trade series. The foreign inflation process, $\pi_{t}^{*}$, is plotted in figure 3 , and the estimates of $\rho_{\pi^{*}}$ and $\sigma_{\pi^{*}}$ capture its persistence of volatility. The remaining sources of cyclical fluctuations are a foreign demand shock, $\hat{y}_{t}^{*}$, and a technology growth shock, $\hat{z}_{t}$. The estimated autocorrelations of these shocks are 0.88 and 0.61 (DSGE) and 0.87 and 0.53 (DSGE-VAR), respectively. In general, we observe that the shock-standard-deviation and autocorrelation estimates obtained with the DSGE-VAR are slightly smaller. The reason is that the DSGE-VAR can capture model misspecification by deviating from cross-equation restrictions, whereas the directly estimated DSGE model has to absorb this misspecification in the exogenous shock processes.

\subsection{The Determinants of Inflation}

This section discusses the impulse responses of the endogenous variables to internal and external shocks. Given that the Central Bank is in an inflation-targeting regime, we focus the discussion on 
the determinants of inflation dynamics. In section 4.4 we showed that the Central Bank seemingly does not respond to exchange rate or terms-of-trade movements. Did this policy manage to insulate the economy, and inflation in particular, from external shocks?

Figure 4 shows the impulse response functions to the five shocks described in section 1: monetary policy, technology, terms of trade, foreign output, and foreign inflation shocks. We overlay two impulse response functions, both of which are computed using the DSGE model. The difference between the two consists in the underlying estimates of the DSGE model parameter: one set of responses is based on the DSGE model estimates, whereas the other reflects the DSGE-VAR estimates. From a qualitative standpoint, the shape of the two response functions is the same. The main difference between them is that the DSGEVAR responses are more pronounced, reflecting the larger estimated standard deviation of shocks documented in table 3.

Monetary policy shocks are contractionary shocks to the feedback rule (equation 2). As the interest rate increases, inflation and output decrease, and the exchange rate appreciates. Notably, the small estimated amount of nominal rigidities implies that the output response is very modest. Positive technology shocks raise output. As in Lubik and Schorfheide (2007), these shocks also raise marginal costs and thereby increase inflation and interest rates. ${ }^{8}$

Improvements in the terms of trade lead to an increase of output and a depreciation of the exchange rate, but they have only a moderate effect on inflation. To understand these responses, it is helpful to substitute the definition of CPI inflation (equation 7) into the policy rule (equation 2). We now have a three-equation system in $\hat{R}_{t}, \hat{\pi}_{H, t}$, and $\hat{y}_{t}$. In this system, shocks to the terms of trade, which are assumed to be exogenous, play essentially the same role as policy shocks. They thus have a similar impact on domestic inflation, $\hat{\pi}_{H, t}$ and $\hat{y}_{t}$, as the monetary policy shocks, but of the opposite sign. An appreciation of the terms of trade therefore leads to an increase in output and domestic inflation. The latter roughly compensates the impact of the appreciation, so that overall inflation, $\hat{\pi}_{t}$, does not move much in the end. Output does move, however, indicating that when a central bank responds to overall rather than domestic inflation, it fails to insulate the real side of the economy from external shocks (see Galí and Monacelli, 2005).

8. Equation (6) shows that marginal costs and detrended output, $\hat{y}_{t}$, move one to one, for given flexible price output. The latter is an exogenous function of foreign output, $y_{t}^{*}$; see expression (4). 


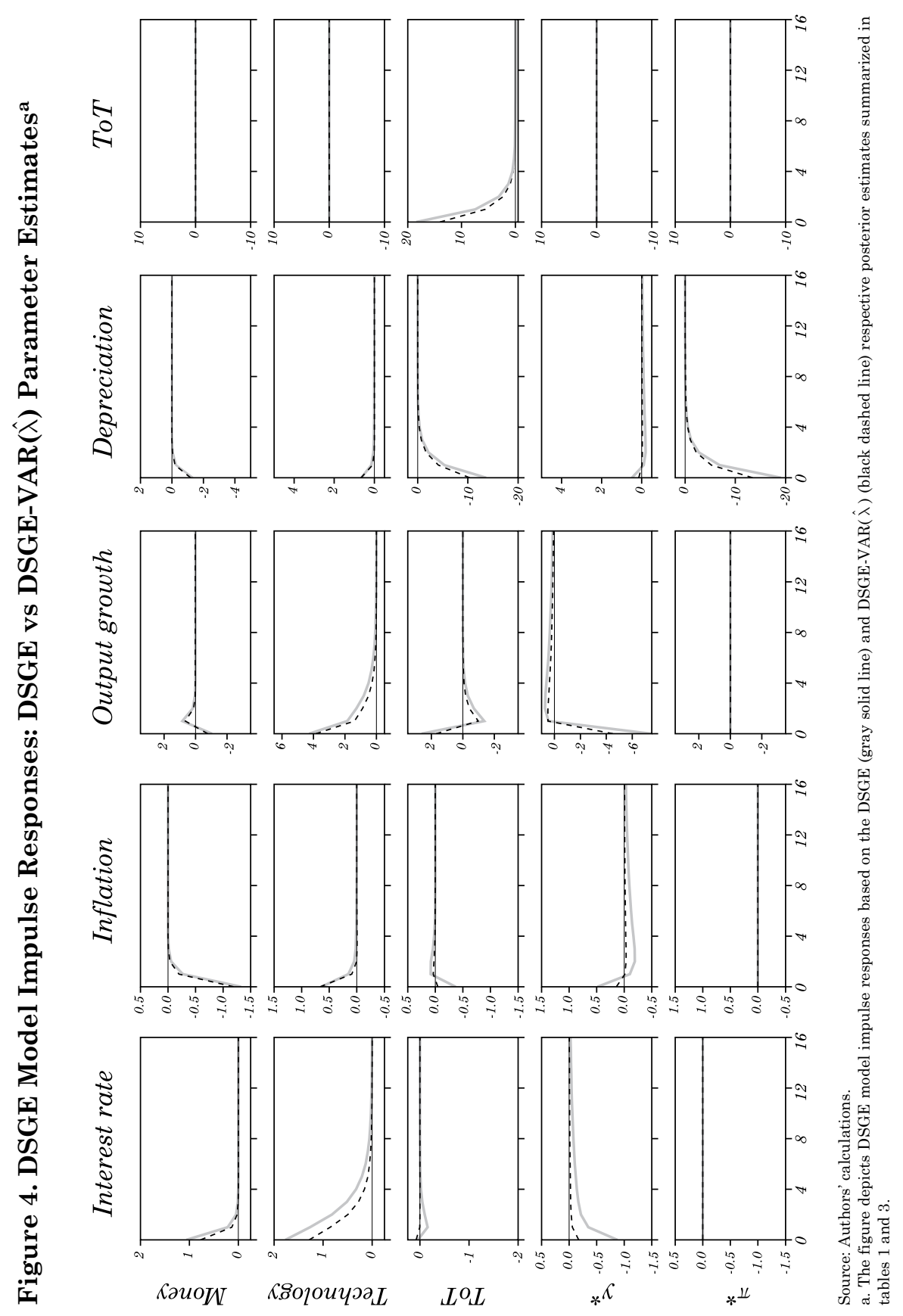


Shocks to foreign output have a negative impact on domestic output, again as in Lubik and Schorfheide (2007). The other variables are not particularly affected. Recall from expression that flexible price output, $\bar{y}_{t}$, depends negatively on foreign output. According to the estimated parameters, the degree of stickiness in this economy is limited, so actual output pretty much behaves as flexible price output. Consequently, inflation is unaffected since marginal costs barely move. Finally, in the baseline specification the Central Bank does not respond to movements in the exchange rate; this isolates the economy from shocks to foreign inflation, which only lead to an appreciation of the currency.

In terms of the determinants of inflation, the interesting feature of figure 4 is that the shocks that move the terms of trade and the nominal exchange rate depreciation-namely, terms-of-trade and foreign inflation shocks-barely affect CPI inflation. According to the DSGE model identification, the shocks that have the largest impact on inflation are largely domestic, namely technology and monetary policy shocks. Notably, these shocks have little effect on the exchange rate depreciation (or on the terms of trade, which are exogenous by construction). In summary, the impulse responses indicate absence of strong comovements between inflation and the external variables. These findings suggest that the monetary authorities have been successful in isolating inflation from foreign disturbances.

It is somewhat surprising that monetary policy shocks have a significant effect on inflation, given that these shocks are avoidable. One possibility is that the Central Bank, in the attempt to respond to future rather than current inflation, makes errors in forecasting inflation. From the model's perspective, these errors appear as policy shocks. Another possible explanation is that the policy reaction function is misspecified, and policy responds to some other variable not included in the reaction function. While this is certainly a possibility, we know from section 4.4 that the missing variable cannot be the exchange rate or the terms of trade.

Figure 4 shows that the impulse responses are generally not very persistent, reflecting the fact that the DSGE model does not generate much internal propagation. Moreover, the DSGE impulse responses are computed under stark identification assumptions, such as exogeneity of the terms of trade. These limitations, as well as the evidence of misspecification discussed in the previous section, suggest that we may want to compare the DSGE model impulse responses to those from the DSGE-VAR and check whether relaxing the cross- 
equation restrictions alters the dynamics substantially. In comparing the DSGE model impulse responses with those from the DSGE-VAR, one should bear in mind that in principle some differences may arise from the fact that the DSGE model does not have an exact finite VAR representation (see Ravenna, 2007, among others). Figure 5 shows that in the case considered here, this is not a quantitatively important issue. The figure compares the DSGE impulse responses with those obtained from the finite order VAR approximation of the DSGE model, that is, $\operatorname{DSGE}-\operatorname{VAR}(\lambda=\infty)$. The two are virtually identical. This implies that if the data were generated by the DSGE model at hand, the DSGEVAR would recover the "true" impulse response functions.

Figure 6 compares the impulse responses computed from the $\operatorname{DSGE}-\operatorname{VAR}(\lambda=\infty)$, which are identical to the DSGE-VAR responses in figure 4 , to those from DSGE-VAR $(\hat{\lambda}) .{ }^{9}$ The figure shows that by and large the differences between the DSGE-VAR $(\lambda=\infty)$ and the DSGE-VAR $(\hat{\lambda})$ impulse responses lie in the dynamics of the nominal exchange rate, which is somewhat more volatile and persistent in the DSGE-VAR than in the DSGE model. In discussing the DSGE model's impulse responses, we remarked that shocks that move inflation do not affect the terms of trade or the exchange rate. This is less the case for the DSGE-VAR $(\hat{\lambda})$, where technology shocks have a substantial impact on inflation and a prolonged effect on the exchange rate. However, compared with the response of exchange rates to terms-oftrade or foreign inflation shocks, the response to technology shocks is small. Hence, the conclusion that inflation has by and large been isolated from external shocks seems to be robust to the presence of misspecification.

The terms-of-trade impulse responses are not very different either. Note that the assumption of exogeneity of the terms of trade is not strictly imposed on the DSGE-VAR. If the data were substantially at odds with this assumption, we would see differences between the two sets of impulse responses in the last column. While we see some differences, these are small relative to the magnitude of movements in the terms of trade. Thus, the short-cut of treating the terms of trade as exogenous in the DSGE model is supported by our empirical analysis. In discussing figure 4, we noted that according to the DSGE model, the economy is isolated from foreign inflation shocks since the Central Bank does not respond to the exchange

9. We do not show the posterior bands for simplicity of exposition; they are available on request. 


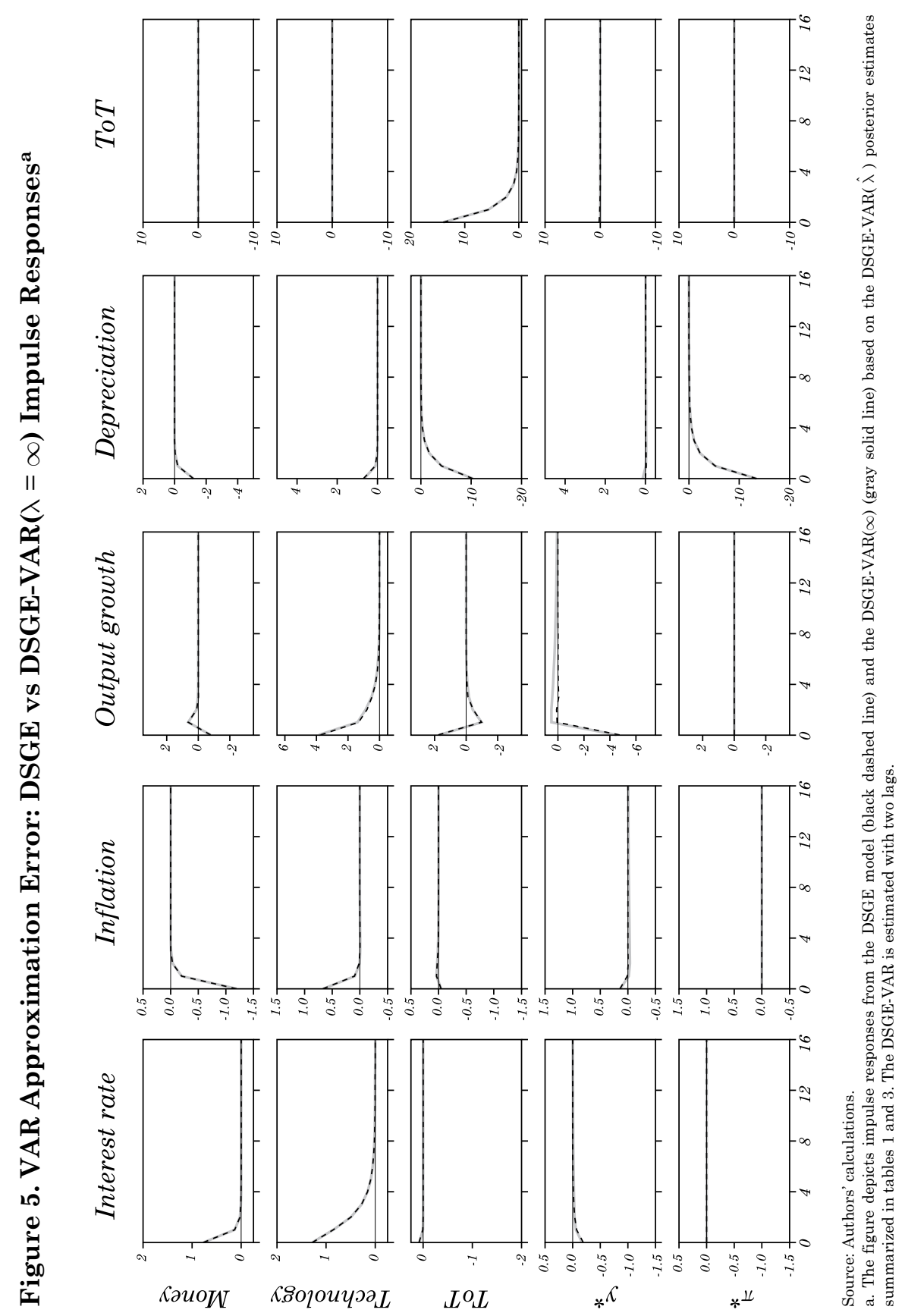




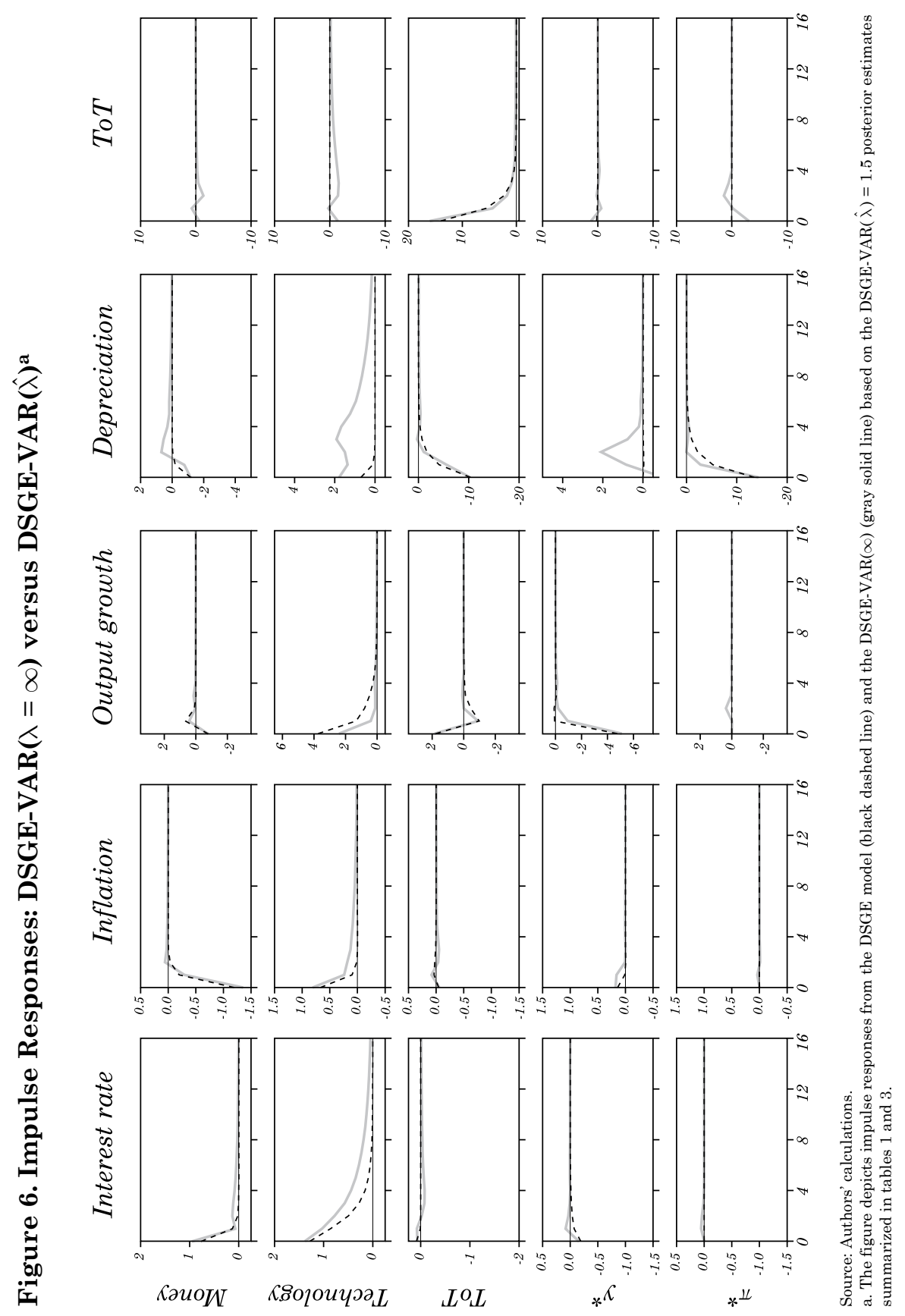


rate. This is still the case according to the DSGE-VAR in figure 6, even though the cross-equation restrictions that deliver this result are not dogmatically imposed.

In summary, figure 6 suggests that the misspecification found in section 4.2 is not very important from an economic standpoint. This result must be interpreted with caution, however. The identification in the DSGE-VAR is, by construction, linked to that in the DSGE model. While this may be a virtue, as it ties the DSGE-VAR impulse responses to those of the underlying DSGE model, it can also be a drawback. There may be other DSGE models, and other identification schemes, that are equally capable of describing the data. By construction, DSGEVAR is not going to be able to uncover such models. Finally, because of the short sample, the data may simply not be informative enough to point out the deficiencies of this model.

\subsection{A Look at Alternative Policy Rules}

In this section, we examine the effect on macroeconomic volatility of responding more or less aggressively to inflation. Conducting this policy analysis with the DSGE model is straightforward. We simply re-solve the model under the new policy rule. Using the DSGE-VAR to assess the effect of changes in the monetary policy rule is conceptually more difficult. We apply the approaches recently proposed in Del Negro and Schorfheide (2008b) to use the DSGEVAR to check the robustness of the DSGE model analysis in view of the misspecification of the structural model that we documented in the previous subsections.

Figure 7 describes how the DSGE model impulse responses change as the parameter $\psi_{1}$ in the policy reaction function varies from 1.25 to 2.75 and to 3.50. Although each plot has three lines, it appears to have only two because raising the reaction to inflation from its estimated value of 2.75 to 3.50 has virtually no impact on the dynamics. Hence, responding more aggressively to inflation would not have any effect on the Chilean economy, at least according to this estimated model. Conversely, a much weaker response to inflation $\left(\psi_{1}=1.25\right)$ would have serious effects, especially on inflation. The response to both technology and monetary policy shocks would be much more pronounced.

Figure 8 shows DSGE-model-based variance differentials with respect to the historical policy rule, $\psi_{1}=2.75$, as we vary $\psi_{1}$ on a grid ranging from 1.00 to 3.50. The figure graphs the posterior mean (90 percent posterior bands) differentials under the DSGE 


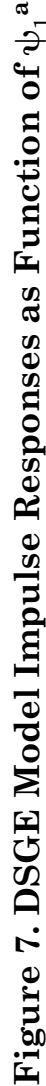
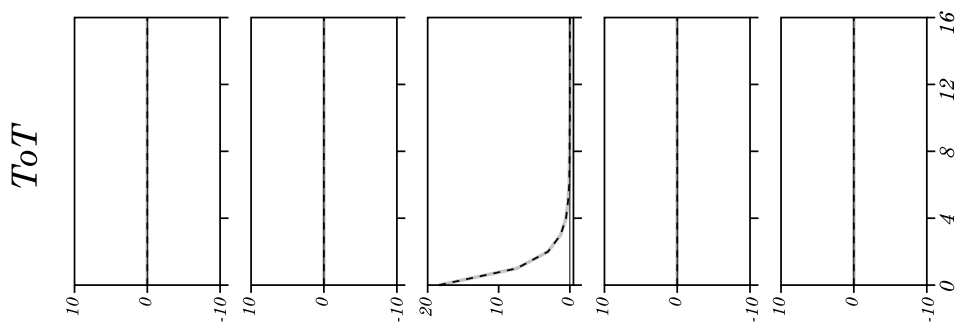

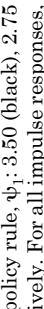
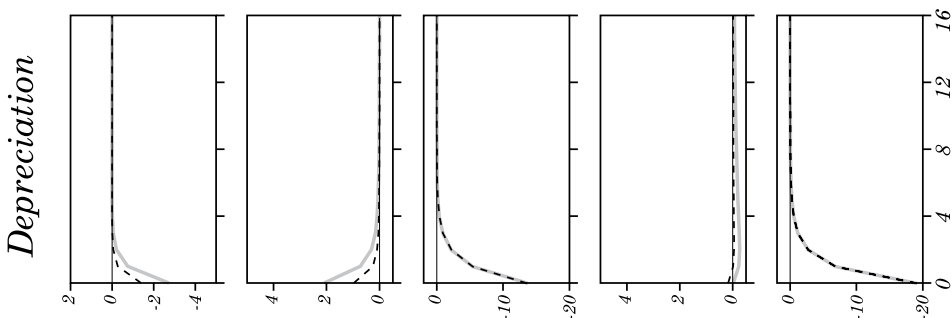

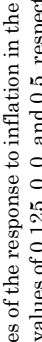

2
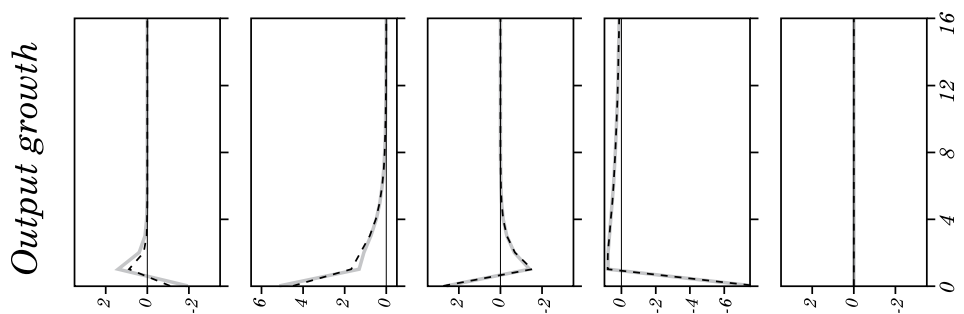

స్
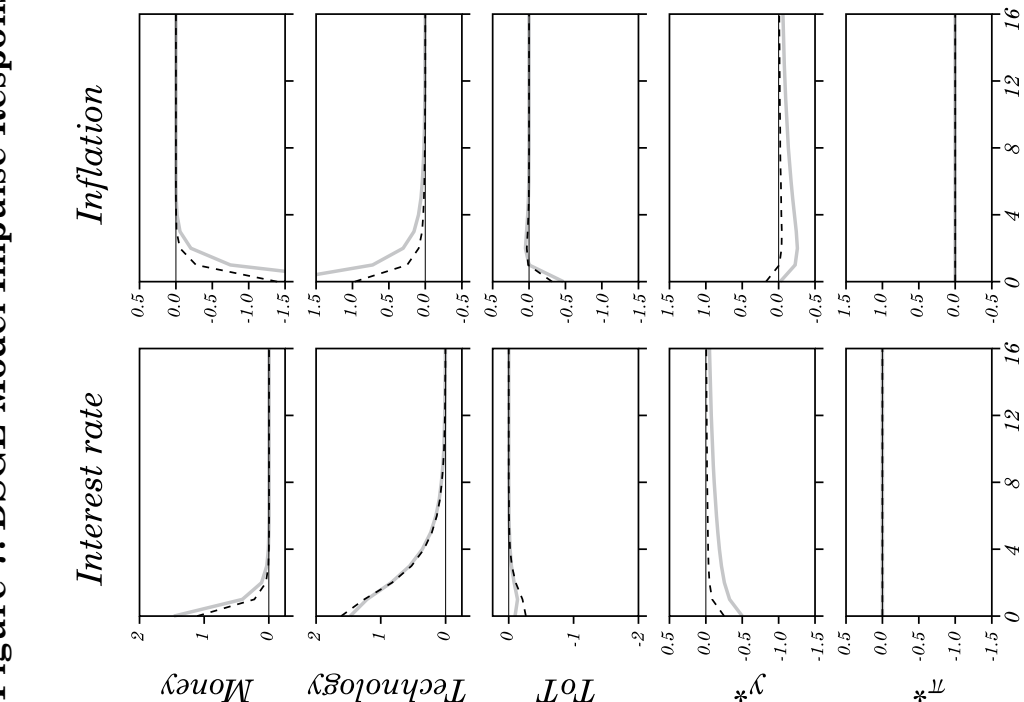

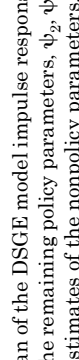

ซึ

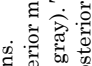

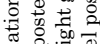

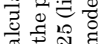

년 을 멈

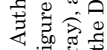

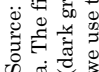


model estimates of parameters (second column of table 3), as well as the posterior mean (90 percent posterior bands) differentials under the DSGE-VAR estimates of parameters (third column of table 3). Consistent with figure 7 , under both sets of estimates the variance of inflation increases substantially as $\psi_{1}$ decreases below 1.50, while not much happens as $\psi_{1}$ increases from 2.75 to 3.50. The magnitude of the increase in the variance differential differs substantially under the two sets of estimates. The shocks are estimated to be more persistent and more variable under the DSGE than under DSGE-VAR, so the effect of changes in policy on the variability of inflation is larger. One can view the higher persistence and variability of the exogenous shocks under the DSGE model estimates as a consequence of the model's misspecification, as discussed in section 4.2 , and therefore not trust the outcomes of the policy analysis exercise under these estimates. In any case, these results highlight the sensitivity of the policy exercises to the estimates of the processes followed by the exogenous shocks, a point made in Del Negro and Schorfheide (2008b).

\section{Figure 8. Comparative Performance of Policy Rules: Benchmark DSGE versus DSGE-VAR $(\lambda=2)$ Parameter Estimates $^{\text {a }}$}

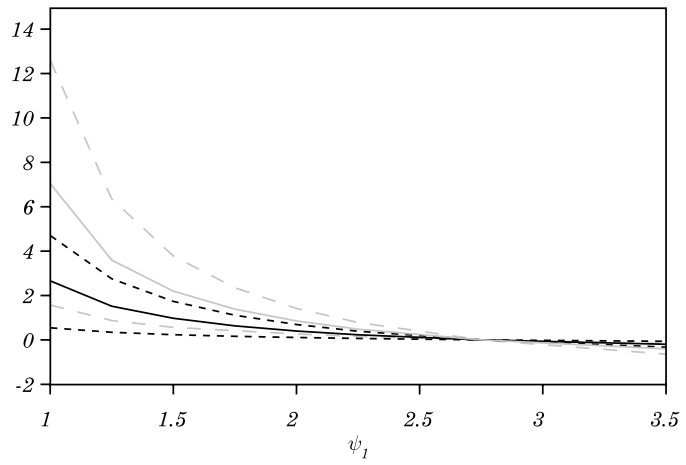

Source: Authors' calculations.

a. Posterior expected variance differentials as a function of $\psi_{1}$ relative to the baseline policy rule, $\psi_{1}=2.75$. The remaining policy parameters, $\psi_{2}, \psi_{3}, \psi_{4}$, and $\rho_{r}$, are kept at the baseline values of $0.125,0,0$, and 0.5 , respectively. Negative differentials signify a variance reduction relative to the baseline rule. Differentials are computed using DSGE-VAR posterior (gray) and DSGE model (black) posterior estimates of the nonpolicy parameters, $\theta_{(n p)}$, summarized in table 3. The solid (dashed) gray lines represent the posterior mean (90 percent posterior bands) differentials under the DSGE model estimates of parameters (second column of table 3). The solid (dotted) black lines represent the posterior mean (90\% posterior bands) differentials under the DSGE-VAR estimates of parameters (third column of table 3). 
Figure 9. Comparative Performance of Policy Rules: DSGE versus DSGE-VAR Policy-Invariant Misspecification and DSGE-VAR Backward-Looking Analysis ${ }^{a}$

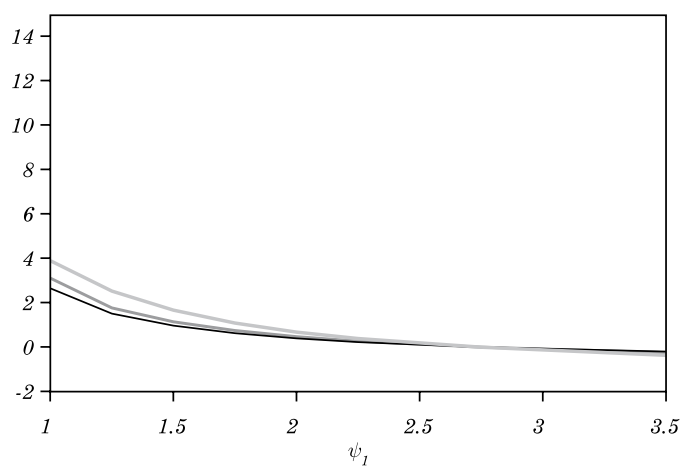

Source: Authors' calculations.

a. Posterior expected variance differentials as a function of $\psi_{1}$ relative to the baseline policy rule, $\psi_{1}=2.75$. The remaining policy parameters, $\psi_{2}, \psi_{3}, \psi_{4}$, and $\rho_{r}$, are kept at the baseline values of $0.125,0,0$, and 0.5 , respectively. Negative differentials signify a variance reduction relative to baseline rule. Differentials are computed using the DSGE-VAR backward-looking analysis (light gray), the DSGE-VAR policy-invariant misspecification scenario (dark gray), and the DSGE model (black), where the latter uses the DSGE-VAR $(\lambda=2)$ posterior estimates of the nonpolicy parameters, $\theta_{(n p)}$, summarized in table 3 .

Figure 9 shows the expected changes in the variability of inflation under three different approaches to performing the policy experiment. Under all three approaches, the experiment is the one just described-that is, varying $\psi_{1}$ in a grid ranging from 1.00 to 3.50. The first approach is the same as in the previous paragraph: it amounts to performing the experiment using the DSGE model under the DSGE-VAR estimates of the nonpolicy parameters. The second approach is called the DSGE-VAR policy-invariant misspecification and is described in detail in Del Negro and Schorfheide (2008b). This approach to policy assumes that while the cross-equation restrictions change with policy, the deviations from the cross-equation restrictions outlined in figure 6 are policy invariant. More specifically in terms of the DSGE-VAR notation, the matrices that embody the crossequation restriction-namely, $\Psi^{*}(\theta)$ and $\boldsymbol{\Sigma}^{*}(\theta)$ —change with $\psi_{1}$, but the deviations—namely, $\Psi^{\Delta}=\Psi-\Psi^{*}(\boldsymbol{\theta})$ and $\boldsymbol{\Sigma}^{\Delta}=\boldsymbol{\Sigma}-\boldsymbol{\Sigma}^{*}(\boldsymbol{\theta})$ - do not. ${ }^{10}$

10. We work with the moving average rather than the VAR representations, as discussed in Del Negro and Schorfheide (2008b). Thus we literally treat the deviations from the DSGE-VAR $(\infty)$ impulse responses in figure 5 as policy invariant. 
This approach may be appealing if one thinks that these deviations capture low or high frequency movements in the data that are not going to be affected by policy. The variance differential under this alternative approach is about the same as under the DSGE model (as are the bands, which we do not show to avoid cluttering the figure). This is not surprising given that the deviations from the cross-equation restrictions are small, particularly for inflation.

The second approach is called the DSGE-VAR backward-looking analysis and is again described in detail in Del Negro and Schorfheide (2008b). Under this approach, the DSGE-VAR is treated as an identified VAR: the change in $\psi_{1}$ only affects the policy rule (Sims, 1999), and it does not affect the remaining equation of the system. Under this approach, the cross-equation restriction are completely ignored. Although the rationale for ignoring the cross-equation restrictions when the deviations are small is questionable, the line in question is not very different from the other two. In the end, we find that in this application the treatment of misspecification leads to rather small differences relative to those shown in figure 8. As in Del Negro and Schorfheide (2008b), inference about the nonpolicy parameters, and in particular about the persistence and standard deviation of the shocks, is key in evaluating the outcomes of different policy rules.

\section{Conclusion}

We estimate the small open economy DSGE model used in Lubik and Schorfheide (2007) on Chilean data for the inflation-targeting period, 1999-2007, using data on the policy rate, inflation, real output growth, nominal exchange depreciation, and log differences in the terms of trade. We also estimate a Bayesian VAR with a prior generated from the small open economy DSGE model, following the DSGE-VAR methodology proposed in Del Negro and Schorfheide (2004, 2008b). The purpose of the DSGE-VAR is to check whether the answers provided by the DSGE model are robust to the presence of misspecification, where misspecification is defined as deviations from the cross-equation restrictions imposed by the model.

Our empirical results can be summarized as follows. First, our estimates of a monetary reaction function indicate that the Central Bank of Chile did not respond significantly to movements in the exchange rate and terms of trade. Second, our DSGE-VAR analysis suggests that, in part because of a short estimation sample, it is helpful to tilt the VAR estimates toward the restrictions generated 
by our small open economy DSGE model. A VAR that is estimated without a tight prior is unlikely to produce good forecasts or sharp policy advice. Third, both our estimated DSGE model and the DSGE-VAR indicate that the observed inflation variability is mostly due to domestic shocks. Moreover, despite the statistical evidence of DSGE model misspecification, the DSGE-VAR's implied dynamic responses to structural shocks closely mimic the DSGE model impulse response functions.

Finally, we find that a stronger Central Bank response to inflation movements would produce little change in inflation volatility, whereas a substantial decrease would lead to a spike in volatility. We obtain a quantitatively similar result if we conduct the policy analysis with the DSGE model. An important caveat to the policy analysis exercise is that the DSGE model used here has many restrictive assumptions, so it may not capture some important policy trade-offs. Nevertheless, we believe that a few lessons can be learned from this exercise, which are likely to carry over to more sophisticated models. First, the outcome of policy experiments is very sensitive to the estimates for the parameters describing the law of motion of the exogenous shocks. Second, the presence of misspecification - that is, the fact that the DSGE model is rejected relative to a more loosely parameterized model—does not necessarily imply that the answers to policy exercises obtained from the DSGE model are not robust. The DSGE-VAR methodology provides ways of checking the robustness of the policy advice under different assumptions about misspecification, and we hope this can be useful in applied work at central banks. 
ApPENDIX

\section{Model Derivation}

Time $t$ decisions are made after observing all current shocks. Variables with the subscript $t-s$, where $\mathrm{s} \geq 0$, are known at time $t$. We assume that asset markets are complete. For each state of nature, there is a security that pays one Chilean peso or one U.S. dollar.

\section{Households}

Domestic households solve the following decision problem

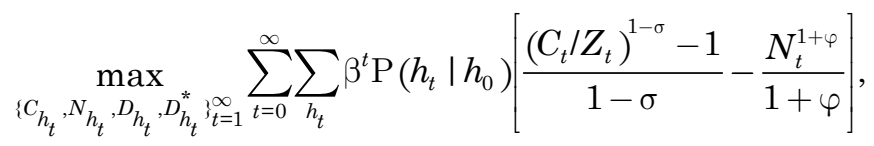

subject to ,

$$
\begin{aligned}
& P_{h_{t}} C_{h_{t}}+\sum_{h_{t+1}}\left(\mathrm{Q}_{h_{t+1} \mid h_{t}} D_{h_{t+1}}+\varepsilon_{h_{t}} \mathrm{Q}_{h_{t+1} \mid h_{t}}^{*} D_{h_{t+1}}^{*}\right) \leq W_{h_{t}} N_{h_{t}} \\
& +D_{h_{t}}+\varepsilon_{h_{t}} D_{h_{t}}^{*}+\int \Omega_{h_{t}}(i) d i
\end{aligned}
$$

where $h_{t+\tau}$ denotes the history of events up to time $t+\tau, \mathrm{P}\left(h_{t+\tau} \mid h_{t}\right)$ is its probability conditional on time $t$ information, $C_{h_{t}}$ is consumption of a composite good in state $h_{t}, N_{h_{t}}$ is hours worked, $P_{h_{t}}$ is the nominal price level of the composite good, $D_{h_{t+\tau}}\left(D_{h_{t+\tau}}^{*}\right)$ is holdings of a security that pays one unit of the domestic currency (foreign currency) in state $h_{t+\tau}, \mathrm{Q}_{h_{t^{+} \tau} \mid h_{t}}\left(\mathrm{Q}_{h_{t^{+} \tau} \mid h_{t}}^{*}\right)$ is its current price in pesos (in state $\left.h_{t}\right), \varepsilon_{h_{t}}$ is the nominal exchange rate (domestic currency/foreign currency), $T_{h_{t}}$ are nominal transfers, and $\Omega_{h_{t}}(i)$ are (nominal) dividends earned from domestic firm $i$. Note that $\sum_{h_{t+1}} \mathrm{Q}_{h_{t+1} \mid h_{t}}=1 / R_{h_{t}}$ is the inverse of the one-period gross nominal risk-free interest rate in pesos, and $\Sigma_{h_{t+1}} \mathrm{Q}_{h_{t+1} \mid h_{t}}^{*}=1 / R_{h_{t}}^{*}$ is the inverse of the one-period gross nominal riskfree interest rate in dollars. Finally, $Z_{t}$ is a world technology process, which is assumed to follow a random walk with drift. From now on, we use $X_{t}$ to denote a variable $X_{h_{t}}$, and $E_{t}$ to denote $\Sigma_{h^{++} \tau} P\left(h_{t+\tau} \mid h_{t}\right)$.

After detrending consumption and nominal wages according to $c_{t}=C_{t} / Z_{t}$ and $w_{t}=W_{t} /\left(P_{t} Z_{t}\right)$, the first-order conditions can be written as

$$
N_{t}^{\varphi}=c_{t}^{-\sigma} w_{t}
$$




$$
c_{t}^{-\sigma}=\beta E_{t}\left[R_{t} c_{t+1}^{-\sigma}\left(z_{t+1} \pi_{t+1}\right)^{-1}\right],
$$

and

$$
0=E_{t}\left[\left(R_{t}-R_{t}^{*} e_{t+1}\right) \frac{c_{t+1}^{-\sigma}}{c_{t}^{-\sigma}}\left(z_{t+1} \pi_{t+1}\right)^{-1}\right],
$$

where $z_{t}=Z_{t} / Z_{t-1}, \pi_{t}=P_{t} / P_{t-1}$ is the gross inflation rate, and $e_{t}=\varepsilon_{t} / \varepsilon_{t-1}$ is the gross depreciation rate.

\section{Terms of Trade and the Real Exchange Rate}

Let $P_{H, t}$ and $P_{F, t}$ be the domestic price of home- and foreignproduced goods, respectively. Define the terms of trade as follows:

$$
Q_{t}=\frac{P_{H, t}}{P_{F, t}}
$$

We assume that the law of one price for foreign goods holds:

$$
P_{F, t}=\varepsilon_{t} P_{F, t}^{*} .
$$

Here $P_{F, t}^{*}$ is the price of the foreign-produced good in the foreign country, measured in foreign currency. We also assume that domestically produced goods have a negligible weight in foreign consumption. Specifically, let $\vartheta$ be the relative size of the domestic economy (defined more precisely below). We define $\alpha_{*}=\vartheta \alpha$ and we let $\vartheta \rightarrow 0$. Hence, $P_{F, t}^{*}$ will be approximately equal to the foreign CPI, $P_{t}^{*}$, and we can express the terms of trade as

$$
Q_{t}=\frac{P_{H, t}}{\left(\varepsilon_{t} P_{t}^{*}\right)}
$$

Both an exchange rate depreciation and foreign inflation reduce the terms of trade- that is, they make imports more expensive. Let $P_{t}$ be the domestic CPI. The real exchange rate is defined as

$$
S_{t}=\frac{\varepsilon_{t} P_{t}^{*}}{P_{t}}
$$


Thus, the relative price $P_{H, t} / P_{t}$ can be expressed as

$$
\frac{P_{H, t}}{P_{t}}=Q_{t} S_{t}
$$

\section{Composite Goods}

There are firms that buy quantities $C_{H, t}$ and $C_{F, t}$ of domesticallyproduced and foreign-produced goods and package them into a composite good that is used for consumption by the households. These firms maximize profits in a perfectly competitive environment:

$$
\max _{C_{t}, C_{H, t}, C_{F, t}} P_{t} C_{t}-P_{H, t} C_{H, t}-P_{F, t} C_{F, t},
$$

subject to $C_{t}=\left[(1-\alpha)^{1 / \eta} C_{H, t}^{\frac{\eta-1}{\eta}}+\alpha^{1 / \eta} C_{F, t}^{\frac{\eta-1}{\eta}}\right]^{\frac{\eta}{\eta-1}}$.

We deduce from the first-order conditions and a zero-profit condition that

$$
\begin{aligned}
& C_{H, t}=(1-\alpha)\left(\frac{P_{H, t}}{P_{t}}\right)^{-\eta} C_{t} ; \quad C_{F, t}=\alpha\left(\frac{P_{F, t}}{P_{t}}\right)^{-\eta} C_{t} ; \\
& P_{t}=\left[(1-\alpha) P_{H, t}^{1-\eta}+\alpha P_{F, t}^{1-\eta}\right]^{\frac{1}{1-\eta}} .
\end{aligned}
$$

Dividing equation (A12) by $P_{t}$ and rearranging terms leads to the following relationship between the real exchange rate and the terms of trade:

$$
S_{t}=\left[(1-\alpha) Q_{t}^{1-\eta}+\alpha\right]^{\frac{1}{\eta-1}} \text {. }
$$

The domestically-produced good, supplied in overall quantity $Y_{t}$, is itself a composite made up of a continuum of domestic intermediate goods, $Y_{t}(i)$ :

$$
Y_{t}=\left[\int_{0}^{1} Y_{t}(i)^{\frac{\mu-1}{\mu}} d i\right]^{\frac{\mu}{\mu-1}} \text {. }
$$


We assume that there are perfectly competitive firms that buy the domestic intermediate goods, package them, and resell the composite good to the firms that aggregate $C_{H, t}$ and $C_{F, t}$. These firms solve the following problem:

$\max _{Y_{t}, Y_{t}(i)} P_{H, t} Y_{t}-\int_{0}^{1} P_{H, t}(i) Y_{t}(i) d i$,

subject to $Y_{t}=\left[\int_{0}^{1} Y_{t}(i)^{\frac{\mu-1}{\mu}} d i\right]^{\frac{\mu}{\mu-1}}$.

The first-order conditions and a zero-profit condition lead to

$Y_{t}(i)=\left(\frac{P_{H, t}(i)}{P_{H, t}}\right)^{-\mu} Y_{t}, \quad P_{H, t}=\left[\int_{0}^{1} P_{H, t}(i)^{1-\mu} d i\right]^{\frac{1}{1-\mu}}$.

\section{Domestic Intermediate Goods}

The producers of the domestic intermediate goods, $Y_{t}(i)$, are monopolistic competitors. Firms can reoptimize prices in each period with probability $1-\theta$. We assume that firms that are unable to reoptimize their price, $P_{H, t}(i)$, will increase according to the steady-state inflation rate, $\pi_{H, *}$. The firms use today's prices of statecontingent securities to discount future nominal profits. The firms' production function is linear in labor:

$Y_{t}(i)=Z_{t} N_{t}(i)$

where productivity, $Z_{t}$, is not firm specific and its growth rate, $z_{t}=Z_{t} / Z_{t-1}$, follows an $\mathrm{AR}(1)$ process:

$\left(\ln z_{t}-\gamma\right)=\rho_{z}\left(\ln z_{t-1}-\gamma\right)+\varepsilon_{t}^{z}$,

where $\gamma$ is the steady-state growth rate of productivity. The firms' problem is given by

$$
\max _{\tilde{P}_{H, t}(i),\left\{Y_{t+\tau}(i)_{\tau}^{\infty} \infty\right.}^{\infty} E_{t}\left[\sum_{\tau=0}^{\infty} \theta^{\tau} Q_{t+\tau \mid t} Y_{t+\tau}(i)\left(\tilde{P}_{H, t}(i) \pi_{H, *}^{\tau}-M C_{t+\tau}^{n}\right)\right],
$$


Subject to $Y_{t+\tau}(i) \leq\left(\frac{\tilde{P}_{H, t}(i) \pi_{H, *}^{\tau}}{P_{H, t+\tau}}\right)^{-\mu} Y_{t+\tau}$,

where $M C_{t+\tau}^{n}=W_{t+\tau} / Z_{t+\tau}$ is the nominal marginal cost and $Q_{t+\tau \mid t}$ is the time $t$ price of a security that pays one unit of domestic currency in period $t+\tau$.

We are considering a symmetric equilibrium in which all firms solve the same problem and we eliminate the index $i$. The firms' firstorder condition can then be written as:

$$
E_{t}\left[\sum_{\tau=0}^{\infty} \theta^{\tau} Q_{t+\tau \mid t}\left(\frac{\tilde{P}_{H, t^{*}} \pi_{,^{*}}^{\tau}}{P_{H, t+\tau}}\right)^{-\mu} Y_{t+\tau}\left[(\mu-1) \tilde{P}_{H, t} \pi_{H, *}^{\tau}-\mu M C_{t+\tau}^{n}\right]\right]=0 .
$$

The fraction of firms that are allowed to reoptimize their price is $1-\theta$, while all others update their price by the steady-state inflation rate. Hence,

$$
P_{H, t}=\left[\theta \tilde{P}_{H, t}^{1-\mu}+(1-\theta)\left(\pi_{H, *} P_{H, t-1}\right)^{1-\mu}\right]^{\frac{1}{1-\mu}} .
$$

We now express both the nominal marginal costs and the price chosen by firms that are able to reoptimize in terms of the price of the domestic good:

$$
m c_{t}=\frac{M C_{t}^{n}}{P_{t}^{H}}=\frac{W_{t}}{Z_{t} P_{t}} \frac{P_{t}}{P_{t}^{H}}=w_{t} Q_{t}^{-1} S_{t}^{-1}
$$

and

$$
\tilde{p}_{H, t}=\frac{\tilde{P}_{H, t}}{P_{H, t}} .
$$

Moreover, in equilibrium

$$
Q_{t+\tau \mid t}=\beta^{\tau} \frac{\lambda_{t+\tau}}{\lambda_{t}}=\beta^{\tau} \frac{c_{t+\tau}^{-\sigma} P_{t} Z_{t}}{c_{t}^{-\sigma} P_{t+\tau} Z_{t+\tau}}
$$


Thus, the optimal pricing rule can be restated as

$$
\tilde{p}_{H, t}=\frac{\mu}{\mu-1} \frac{E_{t}\left[\sum_{\tau=0}^{\infty}\left(\beta \theta^{\tau}\right) \frac{c_{t+\tau}^{-\sigma}}{c_{t}^{-\sigma}}\left(\frac{\pi_{H, *}^{\tau}}{\prod_{s=1}^{\tau} \pi_{H, t+s}}\right)^{-\mu} S_{t+\tau} Q_{t+\tau} y_{t+\tau} m c_{t+\tau} \prod_{s=1}^{\tau} \pi_{H, t+s}\right]}{E_{t}\left[\sum_{\tau=0}^{\infty}\left(\beta \theta^{\tau}\right) \frac{c_{t+\tau}^{-\sigma}}{\left.c_{t}^{-\sigma}\left(\frac{\pi_{H, *}^{\tau}}{\prod_{s=1}^{\tau} \pi_{H, t+s}}\right)^{-\mu} S_{t+\tau} Q_{t+\tau} y_{t+\tau} \pi_{H, *}^{\tau}\right]}\right.} .
$$

Here we used the fact that $P_{H, t} / P_{t}=S_{t} Q_{t}$. We can re-write equation (A21) as

$$
1=\left[\theta \tilde{p}_{H, t}^{1-\mu}+(1-\theta)\left(\pi_{H, *} / \pi_{H, t-1}\right)^{1-\mu}\right]^{\frac{1}{1-\mu}} .
$$

\section{Domestic Market Clearing and Aggregate Production Function}

The market for domestically produced goods clears if the following condition in terms of variables detrended by $Z_{t}$ is satisfied

$y_{t}=c_{H, t}+c_{H, t}^{*}$.

After substituting equation (A11) into equation (A26), we obtain

$$
\begin{aligned}
y_{t} & =c_{H, t}+c_{H, t}^{*}=(1-\alpha)\left(\frac{P_{H, t}}{P_{t}}\right)^{-\eta} c_{t}+\alpha \vartheta\left(\frac{P_{H, t} / \varepsilon_{t}}{P_{t}^{*}}\right)^{-\eta} c_{t}^{*} \\
& =(1-\alpha)\left(S_{t} Q_{t}\right)^{-\eta} c_{t}+\alpha \vartheta Q_{t}^{-\eta} c_{t}^{*} .
\end{aligned}
$$

Finally, the aggregate production function for the domestic economy is given by

$$
y_{t}=N_{t}\left[\int\left(\frac{P_{H, t}(i)}{P_{H, t}}\right)^{-\mu} d i\right]^{-1} \text {. }
$$


The households receive the profits generated by the monopolistically competitive domestic intermediate goods producers. Firm $i$ generates the following profit:

$\Omega_{t}(i)=Y_{t}(i) P_{H, t}(i)-N_{t}(i) W_{t}$

Using the demand function (A16), we can write

$\Omega_{t}(i)=P_{H, t}^{1-\mu} \frac{Y_{t}}{P_{H, t}^{\mu}}-N_{t}(i) W_{t}$.

Integrating both sides and using the expression for the price of the composite good, we obtain

$\int \Omega_{t}(i) d i=P_{H, t} Y_{t}-W_{t} N_{t}$

Finally, we deduce from the budget constraint that

$$
P_{h_{t}} C_{h_{t}}-P_{H, h_{t}} Y_{h_{t}}=D_{h_{t}}+\varepsilon_{h_{t}} D_{h_{t}}^{*}-\sum_{h_{t+1}}\left(\begin{array}{l}
\mathrm{Q}_{h_{t+1} \mid h_{t}} D_{h_{t+1}} \\
+\varepsilon_{h_{t}} \mathrm{Q}_{h_{t+1} \mid h_{t}}^{*} D_{h_{t+1}}^{*}
\end{array}\right) \text {. }
$$

\section{The Rest of the World}

We begin by exploiting the perfect-risk-sharing assumption to obtain a relationship between domestic and foreign consumption:

$$
\left(\frac{c_{t+1}}{c_{t}}\right)^{\sigma} \pi_{t+1}=\left(\frac{c_{t+1}^{*}}{c_{t}^{*}}\right)^{\sigma} \pi_{t+1}^{*} e_{t+1} .
$$

Equation (A.31) can be rewritten as follows:

$$
\left(\frac{c_{t+1}}{c_{t+1}^{*}}\right)^{\sigma} \frac{P_{t+1}}{\varepsilon_{t+1} P_{t+1}^{*}}=\left(\frac{c_{t}}{c_{t}^{*}}\right)^{\sigma} \frac{P_{t}}{\varepsilon_{t} P_{t}^{*}} .
$$

This equation links consumption growth at home and abroad. To obtain implications about the level of consumption in the two countries, we assume that in period $t=0, S_{0}=1$. Moreover, we let $\vartheta=C_{0} / C_{0}^{*}$. We thus deduce that in period $t$ 
$c_{t}=\vartheta c_{t}^{*} S_{t}^{1 / \sigma}$.

We can now rewrite the market-clearing condition for the domestically produced good, recalling that $P_{H, t} / P_{t}=Q_{t} S_{t}$. We substitute equation (A32) into equation (A27) to obtain

$y_{t}=\vartheta c_{t}^{*} Q_{t}^{-\eta}\left[(1-\alpha) S_{t}^{1 / \sigma-\eta}+\alpha\right]$.

In slight abuse of notation, the foreign analog of equation (A30) is

$$
\varepsilon_{h_{t}} P_{h_{t}}^{*} C_{h_{t}}^{*}-\varepsilon_{h_{t}} P_{F, h_{t}}^{*} Y_{h_{t}}^{*}=D_{F, h_{t}}+\varepsilon_{h_{t}} D_{F, h_{t}}^{*}-\sum_{h_{t+1}}\left(\begin{array}{l}
\mathrm{Q}_{h_{t+1} \mid h_{t}} D_{F, h_{t+1}} \\
+\varepsilon_{h_{t}} \mathrm{Q}_{h_{t+1} \mid h_{t}}^{*} D_{F, h_{t+1}}^{*}
\end{array}\right) .
$$

Since all state-contingent securities are in zero net supply, we obtain the following global resource constraint from the budget constraints of the domestic and foreign households:

$c_{t}+S_{t} c_{t}^{*}=Q_{t} S_{t} y_{t}+S_{t} y_{t}^{*}$.

The equilibrium conditions are given by equations (A2), (A3), (A4), (A8), (A9), (A13), (A22), (A24), (A25), (A28), (A32), and (A33). Moreover, we let $\vartheta \rightarrow 0$ and use the approximation $c_{t}^{*}=y_{t}^{*}$. The system can be closed with interest rate feedback rules for the domestic and foreign central banks.

\section{Steady States}

The central banks at home and abroad are determining the steadystate inflation rates, $\pi_{*}$ and $\pi_{*}^{*}$. Moreover, we assume that $S_{0}=S_{*}=1$. The consumption Euler equation implies that the domestic nominal interest rate is $R_{*}=z_{*} \pi_{*} / \beta$. A constant real interest rate implies that the nominal exchange rate depreciation in steady state is $e_{*}=\pi_{*} / \pi_{*}^{*}$. Uncovered interest rate parity determines the foreign nominal rate: $R_{*}^{*}=R_{*} / e_{*}$. The terms of trade are

$$
Q_{*}=\left[\frac{1}{1-\alpha}\left(S_{*}^{\eta-1}-\alpha\right)\right]^{\frac{1}{1-\eta}}=1 \text {. }
$$


Steady state inflation for the domestic good is $\pi_{H, *}=\pi_{*}$. According to the small open economy assumption, $c_{*}^{*}=y_{*}$. Clearing of the domestic goods market requires $y_{*}=\vartheta y_{*}^{*}$. Perfect risk sharing implies $c_{*}=\vartheta c_{*}^{*}$. The supply-side condition for the domestic good determines the steady-state labor input $N_{*}=y_{*}$. Finally, we can determine $y_{*}$ from the marginal cost condition:

$y_{*}=\left(\frac{\mu-1}{\mu}\right)^{\frac{1}{\varphi+\sigma}}$.

Now the global resource constraint (A34) is indeed satisfied for $Q_{*}=1$.

\section{Log-linearizations}

We use the notation $\hat{X}_{t}$ to denote deviations of a variable $X_{t}$ from its steady state, $X_{*}: \hat{X}_{t}=\ln X_{t} / X_{*}$. The relationship between the real exchange rate and the terms of trade is given by

$$
\hat{S}_{t}=-(1-\alpha) \hat{Q}_{t}
$$

Nominal exchange rates evolve according to

$$
\hat{e}_{t}=\Delta \hat{S}_{t}+\hat{\pi}_{t}-\hat{\pi}_{t}^{*}=-(1-\alpha) \hat{q}_{t}+\hat{\pi}_{t}-\hat{\pi}_{t}^{*}
$$

where $\hat{q}_{t}=\Delta \hat{Q}_{t}$. Inflation in the relative price of the domestic good is given by

$$
\hat{\pi}_{H, t}=\hat{\pi}_{t}+\alpha \hat{q}_{t}
$$

We can use the market-clearing condition for the domestically produced good to determine the level of the terms of trade:

$$
\hat{Q}_{t}=-\frac{1}{\eta+(1-\alpha)^{2}(1 / \sigma-\eta)}\left(\hat{y}_{t}-\hat{y}_{t}^{*}\right)
$$


From perfect risk sharing and the market-clearing condition for the foreign good, we have

$$
\hat{c}_{t}=\hat{c}_{t}^{*}+\frac{1}{\sigma} \hat{S}_{t}=\hat{y}_{t}^{*}-\frac{(1-\alpha)}{\sigma} \hat{Q}_{t}
$$

where we substituted for $S_{t}$ using equation (A35). We can now write the marginal costs as

$$
\widehat{m c}_{t}=\varphi \hat{y}_{t}+\sigma \hat{y}_{t}^{*}+\frac{1}{\eta+(1-\alpha)^{2}(1 / \sigma-\eta)}\left(\hat{y}_{t}-\hat{y}_{t}^{*}\right) .
$$

Define $\kappa=(1-\theta \beta)(1-\theta) / \theta$. Marginal costs determine the inflation of the domestically produced goods via the following Phillips curve:

$$
\hat{\pi}_{H, t}=\beta E_{t}\left[\hat{\pi}_{H, t+1}\right]+\kappa \widehat{m c}_{t} .
$$

The consumption Euler equation is of the form

$$
\hat{c}_{t}=E_{t}\left[\hat{c}_{t+1}\right]-\frac{1}{\sigma}\left(R_{t}-E_{t}\left[\hat{\pi}_{t+1}+\hat{z}_{t+1}\right]\right)
$$

Moreover, by combining the market-clearing condition for the domestic good with the perfect-risk-sharing condition, we deduce that

$$
\hat{y}_{t}=\hat{c}_{t}-\alpha\left[\eta+(1-\alpha)\left(\eta-\frac{1}{\sigma}\right)\right] \hat{Q}_{t} .
$$

Equations (A35) to (A42) determine the evolution of $\hat{Q}_{t}, \hat{S}_{t}, \hat{e}_{t}$, $\hat{\pi}_{t}, \hat{\pi}_{H, t}, \widehat{m c}_{t}, \hat{c}_{t}$, and $\hat{y}_{t}$. We treat $\hat{\pi}_{t}^{*}$ and $\hat{y}_{t}^{*}$ as exogenous and close the system with an interest rate feedback rule that determines $\hat{R}_{t}$.

In section 1 of the main text, we consider a version of the open economy model in which $\varphi=0, \eta=1$, and $1 / \sigma=\tau$. Notice that

$$
\eta+(1-\alpha)^{2}\left(\frac{1}{\sigma}-\eta\right)=\tau+\alpha(2-\alpha)(1-\tau)=\tau+\lambda,
$$

where $\lambda=\alpha(2-\alpha)(1-\tau)$. 
We begin by determining the level of output $\bar{y}_{t}$ in the absence of nominal rigidities, which has to satisfy

$0=\widehat{m c}_{t}=\frac{1}{\tau} \hat{y}_{t}^{*}+\frac{1}{\tau+\lambda}\left(\bar{y}_{t}-\hat{y}_{t}^{*}\right)$.

We deduce that

$\bar{y}_{t}=-\frac{\lambda}{\tau} \hat{y}_{t}^{*}$.

The marginal costs satisfy

$\widehat{m c}_{t}=\frac{1}{\tau+\lambda}\left(\hat{y}_{t}-\bar{y}_{t}\right)$,

which leads to the Phillips curve (equation 5) reported in the main text:

$\hat{\pi}_{H, t}=\beta E_{t}\left[\hat{\pi}_{H, t+1}\right]+\frac{\kappa}{\tau+\lambda}\left(\hat{y}_{t}-\bar{y}_{t}\right)$.

We now manipulate the Euler equation. Using equation (A42), replacing CPI inflation by $\hat{\pi}_{H, t}-\alpha \Delta \hat{Q}_{t}$, and plugging in equation (A38), we can rewrite the consumption Euler equation as

$\hat{y}_{t}-\bar{y}_{t}=E_{t}\left[\hat{y}_{t+1}-\bar{y}_{t+1}\right]-(\tau+\lambda)\left(\hat{R}_{t}-E_{t}\left[\hat{\pi}_{H, t+1}+\hat{z}_{t+1}\right]\right)$,

which is equation in the main text. 


\section{REFERENCES}

Caputo, R. and F. Liendo. 2005. "Monetary Policy, Exchange Rate, and Inflation Inertia in Chile: A Structural Approach.” Working paper 352. Santiago: Central Bank of Chile.

Caputo, R., F. Liendo and J.P. Medina. 2007. "New Keynesian Models for Chile in the Inflation-Targeting Period: A Structural Investigation." In Monetary Policy under Inflation Targeting, edited by F. Mishkin and K. Schmidt-Hebbel. Santiago: Central Bank of Chile.

Céspedes, L.F. and C. Soto. 2007. "Credibility and Inflation Targeting in Chile." In Monetary Policy under Inflation Targeting, edited by F. Mishkin and K. Schmidt-Hebbel. Santiago: Central Bank of Chile.

Christiano, L.J., M. Eichenbaum and C. Evans. 2005. "Nominal Rigidities and the Dynamic Effects of a Shock to Monetary Policy." Journal of Political Economy 113(1): 1-45.

Chumacero, R. 2005. "A Toolkit for Analyzing Alternative Policies in the Chilean Economy." In General Equilibrium Models for the Chilean Economy, edited by R. Chumacero and K. Schmidt-Hebbel. Santiago: Central Bank of Chile.

Del Negro, M. and F. Schorfheide. 2004. "Priors from General Equilibrium Models for VARs." International Economic Review 45(2): 643-73.

- 2008a. "Forming Priors for DSGE Models. and How It Affects the Assessment of Nominal Rigidities." Working paper 13741. Cambridge, Mass.: National Bureau of Economic Research.

. 2008b. "Monetary Policy Analysis with Potentially Misspecified Models." American Economic Review. (forthcoming).

Del Negro, M., F. Schorfheide, F. Smets and R. Wouters. 2007. "On the Fit of New Keynesian Models." Journal of Business and Economics Statistics 25(2): 123-43.

Galí, J. and T. Monacelli. 2005. "Monetary Policy and Exchange Rate Volatility in a Small Open Economy." Review of Economic Studies 72(3): 707-34.

Lubik, T. and F. Schorfheide. 2006. "A Bayesian Look at New Open Economy Macroeconomics." In NBER Macroeconomics Annual 2005, edited by M. Gertler and K. Rogoff. MIT Press.

. 2007. "Do Central Banks Respond to Exchange Rate Movements? A Structural Investigation." Journal of Monetary Economics 54(4): 1069-87. 
Mishkin, F. and K. Schmidt-Hebbel, eds. 2007. Monetary Policy under Inflation Targeting. Santiago: Central Bank of Chile.

Ravenna, F. 2007. "Vector Autoregressions and Reduced-Form Representations of DSGE Models." Journal of Monetary Economics 54(7): 2048-64.

Sims, C.A. 1999. "The Role of Interest Rate Policy in the Generation and Propagation of Business Cycles: What Has Changed since the 30s?" In Beyond Shocks: What Causes Business Cycles?, edited by Jeffrey C. Fuhrer and Scott Schuh. Federal Reserve Bank of Boston.

_. 2002. "Solving Rational Expectation Models." Computational Economics 20(1-2): 1-20.

Smets, F. and R. Wouters. 2003. "An Estimated Stochastic Dynamic General Equilibrium Model of the Euro Area." Journal of the European Economic Association 1(5): 1123-75. 